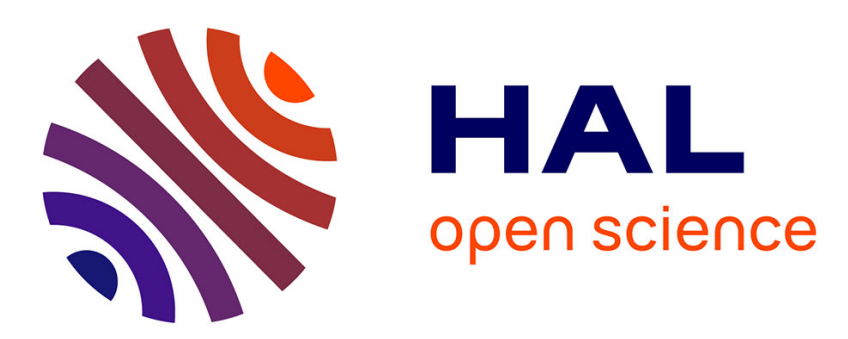

\title{
High resolution simulation of the South Asian monsoon using a variable resolution global climate model
}

\author{
T. P. Sabin, R. Krishnan, Josefine Ghattas, Sébastien Denvil, Jean-Louis \\ Dufresne, Frédéric Hourdin, Pascal Terray
}

\section{- To cite this version:}

T. P. Sabin, R. Krishnan, Josefine Ghattas, Sébastien Denvil, Jean-Louis Dufresne, et al.. High resolution simulation of the South Asian monsoon using a variable resolution global climate model. Climate Dynamics, 2013, 41, pp.173-194. 10.1007/S00382-012-1658-8 . hal-00871962

\section{HAL Id: hal-00871962 \\ https://hal.science/hal-00871962}

Submitted on 22 Jun 2016

HAL is a multi-disciplinary open access archive for the deposit and dissemination of scientific research documents, whether they are published or not. The documents may come from teaching and research institutions in France or abroad, or from public or private research centers.
L'archive ouverte pluridisciplinaire HAL, est destinée au dépôt et à la diffusion de documents scientifiques de niveau recherche, publiés ou non, émanant des établissements d'enseignement et de recherche français ou étrangers, des laboratoires publics ou privés. 


\title{
High resolution simulation of the South Asian monsoon using a variable resolution global climate model
}

T.P Sabin ${ }^{1}$, R. Krishnan ${ }^{1 *}$, Josefine Ghattas ${ }^{2}$, Sebastien Denvil ${ }^{2}$, Jean-Louis Dufresne ${ }^{2}$, Frederic

\author{
Hourdin $^{2}$ and Terray Pascal ${ }^{3}$ \\ ${ }^{1}$ Indian Institute of Tropical Meteorology, Pune, India \\ *krish@tropmet.res.in \\ ${ }^{2}$ Laboratoire Meteorologie Dynamique, IPSL, Paris, France \\ ${ }^{3}$ LOCEAN, IPSL, Paris, France
}

Corresponding author

Dr. R. Krishnan

Centre for Climate Change Research

Indian Institute of Tropical Meteorology, Pune, India

Email : krish@tropmet.res.in 
Abstract: Variable resolution global General Circulation Models (GCMs) with enhanced resolution over the region of interest are an established approach to regional climate downscaling. In this study we have carried out high resolution simulations $(\sim 35 \mathrm{~km})$ over the South Asian monsoon region using the LMDZ (Laboratoire Meteorologie Dynamique and Z stands for zoom) model. Two sets of ten member realizations are produced with and without zooming over the Monsoon region. The simulations without zoom correspond to a global model on a uniform $1^{\circ} \times 1^{\circ}$ grid with the same number of global grid points as those with the stretched grid outside the region of interest. The use of these finer and coarser resolution ensemble members allows us to examine the impact of resolution on the overall quality of the simulated regional monsoon fields. It is found that the monsoon simulation with high-resolution zooming greatly improves the representation of heavy precipitation along the narrow orography of the Western Ghats Mountains, the northeastern mountain slopes and northern Bay of Bengal (BOB). A realistic Monsoon Trough (MT) is also simulated in the zoomed version, together with a remarkable improvement in representing the associated precipitation and circulation features, as well as the largescale organization of meso-scale convective systems over the MT region. In addition, a more realistic simulation of the monsoon synoptic disturbances (lows and disturbances) along the MT is noted in the high-resolution zoomed simulation. On the other hand, the no-zoom version has limitations in capturing the depressions and their movement, so that the MT zone is relatively dry in this case. Overall, the results from this work demonstrate the usefulness of the high-resolution variable resolution LMDZ model in realistically capturing the interactions among the monsoon large-scale dynamics, the synoptic systems and the meso-scale convective systems, which are essential elements of the South Asian monsoon system. 


\section{Introduction}

The South Asian Monsoon (SAM) circulation, which is a major component of the global climate system, arises primarily from the setting up of a meridional land-sea thermal contrast between the elevated Tibetan Plateau and the tropical Indian Ocean during the boreal summer. Once set up, the SAM circulation is maintained primarily through feedbacks between the large-scale monsoonal flow and the release of latent heat of condensation by moist convective processes (see Krishnamurti and Surgi, 1987). The monsoon rainfall over the region exhibits heterogeneous variations in space and time, which involve interactions among multiple scales of motion (ie., planetary, regional, synoptic, meso and cumulus scales). The accuracy of the SAM rainfall simulations depends heavily on the ability of climate models to realistically capture the interactions among these different scales. Gadgil and Sajani (1998) carried out a detailed analysis of monsoon precipitation simulated by more than thirty models that participated in the Atmospheric Model Intercomparison Project (AMIP: Gates, 1992). They found that a large number of models simulated exceptionally high precipitation over the equatorial Indian Ocean and low rainfall over the Indian subcontinent. Moreover, most models simulated the narrow north-south oriented precipitation band along the Western Ghats as a broad region extending too much to the Arabian Sea and failed to capture the rain shadow over southeast India. These limitations of Atmospheric General Circulation Models (AGCMs) in capturing the monsoon rainfall distribution arise partly due to the coarse resolution of AGCMs and partly due to deficiencies in the model treatment of physical processes like moist-convection, boundary layer fluxes, radiative effects, etc.

Very high resolution global GCMs (eg., the Meteorological Research Institute model from Japan with $20-\mathrm{km}$ horizontal resolution) have been fairly successful in resolving the SAM orographic precipitation maxima along narrow mountains of the Western Ghats and Myanmar (eg., Rajendran and 
Kitoh, 2008, Kitoh and Kusunoki, 2009, Krishnan et al. 2012, Rajendran et al. 2012). However, conducting ensembles of long climate simulations using such high-resolution AGCMs remains a major challenge because of the huge computational power requirements. While high-resolution Regional Climate Models (RCMs) are computationally less expensive and have the ability to resolve finer scale orographic precipitation, they require specification of lateral boundary conditions which inhibits them from providing self-consistent interactions between the global and regional scales of motion (FoxRabinovitz et al. 2006).

Over the years, the use of variable resolution AGCMs have proven to be efficient for regional climate downscaling and analyses of meso-scale and finer features. Various climate modeling groups from Australia, France, United States and Canada, among others, have adopted variable resolution stretched-grid GCMs for regional studies (eg., McGregor, 1996, Zhou and Li 2002, Hourdin et al. 2006, Fox-Rabinovitz et al. 2006). Variable resolution AGCMs do not require any lateral boundary conditions/forcing, avoiding the associated undesirable computational problems. They provide a consistent description of the 2-way interactions between global and regional scales, even if these interactions can be in part altered due to the change of resolution if compared to a high-resolution global model.

The present study addresses the feasibility of using variable resolution AGCMs to understand regional aspects of the South Asian monsoon rainfall, the large-scale organization of monsoon convection / precipitation over the Indian subcontinent and the interactions between monsoon circulation and precipitation. Previous studies based on RCM simulations indicate the potential for improving the spatial distribution of mean monsoon rainfall over South Asia through increased horizontal resolution (e.g., Bhaskaran et al. 1996, Jacob and Podzum 1997, Vernekar and Ji 1999, Lee 
and Suh 2000, Dash et al. 2006). The requirement of specifying lateral boundary conditions for RCM simulations poses restrictions in understanding the interactions between the large-scale summer monsoon circulation and the precipitation distribution over the South Asian region. For example, the monsoon rainfall activity over the Indo-Gangetic plains is closely related to the position and intensity of the Monsoon Trough (MT), as well as the strength of the large-scale southwesterly monsoon flow and the vigor of monsoon convection over the subcontinent (eg., Rao 1976, Alexander et al. 1978, Das 1986, Krishnamurti and Bhalme, 1976, Krishnamurti and Surgi, 1987, Goswami et al., 2003, Joseph and Sabin 2008, Rajeevan et al. 2010, Choudhury and Krishnan, 2011). Likewise, breaks in the monsoon rainfall over central India are characterized by a northward shift of the MT and heavy rainfall over the Himalayan foothills, and involve large-scale circulation anomalies such as the southward intrusions of mid-latitude westerly troughs into the Indo-Pak region, the formation of a blocking ridge over East Asia and the generation of circumglobal teleconnection patterns (e.g. Ramaswamy, 1962, Ramamurthy, 1969, Keshavamurty and Awade, 1974, Raman and Rao, 1981, Krishnan et al. 2000, 2009, Ding and Wang, 2007).

In order to address some of those issues of scale-interactions and the need for high resolution modeling in the SAM region, we designed a specific grid configuration using the variable resolution stretched-grid GCM developed at Laboratoire de Meteorologie Dynamique (LMD), France. The global stretched-grid GCM (LMDZ) used in this study has a high-resolution telescopic zooming over the South Asian region of roughly ( $35 \mathrm{~km}$ longitude x $35 \mathrm{~km}$ latitude), with coarser resolution elsewhere. Given that high resolution GCMs require tremendous computational resources, the use of a global stretched-grid GCM with high-resolution zooming over the SAM region is not only a dynamically and physically consistent approach to regional monsoon downscaling, but it also provides a computationally pragmatic way to address high-resolution monsoon modeling. The present study is 
organized as follows. Section 2 provides a brief description of the LMDZ including the design of numerical experiments and the different datasets used. Section 3 deals with an evaluation of the SAM in the LMDZ model simulations with and without telescopic zooming over the region. Improvements in various aspects of monsoon simulation through telescopic zooming are presented in section 4 . The summary and conclusions of this work are presented in section 5.

\section{Model description, experimental design and datasets}

The model used in this study is LMDZ4, which was developed at Laboratoire de Meteorologie Dynamique, France. The grid is stretchable, so that the model can be used for regional climate studies (Hourdin et al. 2006, Zhou and Li 2002). Moist convection is parameterized in the model based on the Emanuel (1993) scheme. Hourdin et al. (2006) have provided detailed information about the physical processes in the LMDZ model, and a preliminary assessment of the model performance at the global scale. By activating the zoom function, LMDZ4 can be run with very high resolution over the region of interest. The model is driven by prescribed sea surface temperature (SST) as lower-boundary conditions. Being a global model, there is no need for specifying lateral boundary conditions in LMDZ4.

We compare two versions of the models, both based on a global grid made of 360 points in longitude, 180 points in latitude, and 19 hybrid layers in the vertical. In the first "no-zoom" configuration, the grid points are regularly spread in both longitude and latitude. For the second "zoom" configuration, the grid is refined over a large region around India. The zoom is centred at $15^{\circ} \mathrm{N}, 80^{\circ} \mathrm{E}$ and the employed model grid is shown in Fig. 1. The grid-size in the shaded region (Eq$40^{\circ} \mathrm{N}, 45^{\circ} \mathrm{E}-110^{\circ} \mathrm{E}$ ) in Fig. $1 \mathrm{a}$ is less than $35 \mathrm{~km}$. The resolution becomes gradually coarser outside the 
zoom domain. Figure.1a shows the horizontal grid spacing in $\mathrm{km}$ for the present LMDZ4 setup. Over the South Asian region, the dark green grids correspond to a horizontal spacing of about $35 \mathrm{~km}$. As we proceed away from the South Asian region, it can be seen that the grid spacing gradually increases implying decrease of horizontal resolution. Figure.1b shows the distribution of topography and model grids over the South Asian region. It can be seen that the $35 \mathrm{~km}$ grid resolution adequately resolves the narrow mountains along the Western Ghats of India and the west coast of Myanmar which receive very heavy monsoonal rains during boreal summer. In addition, one can notice that the Hindukush mountain range, stretching between central Afghanistan and northern Pakistan, of South-Central Asia is well resolved in the $35 \mathrm{~km}$ model. The importance of resolving these relatively smaller mountains can have significant influence on the moist processes over north-central India during the summer monsoon season, as will be seen later.

For both the zoom and no-zoom model configurations, a twin set of 10 member ensemble runs was performed with the LMDZ4 model. In both cases, we have used the seasonally varying climatological mean observed SST as boundary forcing from the Met Office Hadley Centre (Rayner et al. 2003). The 10 member ensemble runs are started from 10 perturbed initial conditions of 01 January and each simulation goes through end of December ${ }^{1}$. All members use the same seasonally varying climatological SST as boundary condition. For validating the model simulations, different

\footnotetext{
${ }^{1}$ Starting from the ECMWF climatological initial condition of 01 January, the 10 perturbed initial conditions were created by making ten 1 -yr model runs with interannually varying SSTs $(2000-2009)$ as boundary conditions. The model dumps generated after 1 year of integration from the above 10 cases constitute the 10 perturbed initial conditions. It must be mentioned that interannually varying SSTs have been used only for the purpose of creating the perturbed initial conditions. Once the model dumps are generated, the Zoom and No-Zoom ensemble simulations are performed using the seasonally varying climatological SST boundary forcing.
} 
observational datasets have been used. These include the daily gridded rainfall data from India Meteorological Department (Rajeevan et.al., 2006) which is available in $1^{\circ} \mathrm{x} 1^{\circ}$ latitude-longitude grid over India for the period (1951-2007). The monthly gridded rainfall data from the Global Precipitation Climatology Project (GPCP) Version 2 data (Adler et al. 2003) have also been used to evaluate the model's performance and assess the global precipitation pattern. Observed surface temperature data from the Climate Research Unit (CRU) is also utilized for model validation. The simulated atmospheric circulation, mean sea level pressure, specific humidity fields are validated against the European Centre for Medium-Range Weather Forecasts (ECMWF) Re-Analysis Interim (ERA-Interim; Simmons et al. 2006) data for the period 1989-2008.

\section{Simulation of global and SAM regional features with and without telescopic zooming}

In this section, we shall investigate the fidelity of LMDZ model in simulating the observed features of climatological mean circulation during boreal summer.

\subsection{Mean global rainfall and circulation features}

Figure. 2 shows the spatial distribution of seasonal rainfall for the June-July-August-September (JJAS) months from observations and GCM simulations with and without zoom. The simulation of tropical rainfall climatology has proven to be a rather difficult test for current GCMs. Systematic errors in simulating the JJAS mean precipitation can be noted over the Northern region of South America where the model climate is too dry compared to the observed precipitation. Both the zoom and no-zoom versions capture the main features of the global scale distribution of precipitation associated with the South Pacific Convergence Zone, the Asian and African summer monsoons. Both versions overestimate the rainfall over equatorial and tropical West Pacific as compared to GPCP observations. The simulated monsoon rainfall over South Asia is significantly closer to observations in the zoom 
version as compared to the no-zoom case. This point will be discussed in detail in the next section. The pattern correlation between the simulated and observed precipitation climatology in the tropics (0-360 and $35 \mathrm{~S}-35 \mathrm{~N}$ ) is 0.85 for the zoom simulation and 0.81 for the no-zoom simulation.

The JJAS mean circulation at $850 \mathrm{hPa}$ simulated by the zoom and no-zoom experiments are compared with the ERA-Interim reanalysis in Fig.3. Both simulations capture the major general circulation features such as the easterly trades, Inter Tropical Convergence Zones (ITCZ) and the subtropical anticyclones over both the hemispheres of the Pacific and Atlantic Oceans. Other noteworthy features in the simulations include the subtropical anticyclones over the Mascarene and Australian regions in the southern hemisphere ( $\mathrm{SH}$ ) and over Arabia and northern Africa in the NH; the summer monsoon cross-equatorial flow over the Indian Ocean and the convergence of Pacific easterly trades and the southwesterly monsoonal winds near Philippines. It is interesting that the zoom version shows a well-defined cyclonic circulation over the MT region along the Indo-Gangetic plains. Figure.3d shows the latitudinal variation of the zonally averaged zonal winds at $850 \mathrm{hPa}$ from the ERA Interim reanalysis, the zoom and no-zoom simulations. The correlations between the observed and simulated zonally averaged zonal winds at $850 \mathrm{hPa}$ are 0.97 for the zoom simulation and 0.95 for the no-zoom simulation.

The simulated and observed upper tropospheric circulation are presented in Fig.4. The predominant boreal summer upper-tropospheric features which include the Tibetan anticyclone with ridge axis around $25^{\circ} \mathrm{N}$ and the tropical easterly jet-stream (e.g., Koteswaram, 1958, Krishnamurti, 1973, Raghavan, 1973) and the Asian Jet with strong westerlies $\left(>30 \mathrm{~ms}^{-1}\right)$ on the poleward side of the Tibetan anticyclone (see Enomoto et al. 2003, Krishnan et al. 2009) are captured in both the simulations. Notice that the divergent outflow from the Tibetan anticyclone and upper-tropospheric 
cross-equatorial winds is more conspicuous in the zoom simulation as compared to the no-zoom case. The Asian Jet exhibits a wavy structure in the zoom simulation, while it is more zonal in the no-zoom case. Krishnamurti (1971) provided the first observational evidence for planetary scale east-west divergent circulations during the northern summer. He suggested that these thermally direct east-west circulations were associated with mass "spillover" from the intense energy source located over the SAM region. Basically, the upper tropospheric mid-oceanic troughs over the Pacific and Atlantic Oceans in Fig.4 correspond to the descending branches of the tropical east-west circulations during northern summer (see Krishnamurti, 1971). The zoom version shows a trough-like feature in the upper-troposphere over the Mediterranean region (Fig.4b) indicative of subsidence and low-level anticyclonic circulation (Fig.3b) over the region. Rodwell and Hoskins (1996) pointed out that the summertime descent and aridity over the Mediterranean and Eastern Sahara arises due to a Rossby wave response induced by the South Asian monsoon heating. The latitudinal variation of the zonally averaged upper-tropospheric zonal winds from the two simulations and the ERA reanalysis is shown in

Fig.4d. The correlations between the observed and simulated zonally averaged zonal winds at $200 \mathrm{hPa}$ are 0.99 for the zoom simulation and 0.98 for the no-zoom simulation. Based on the analysis described in Figs.2-4, it can be seen that the zoom simulation preserves the realism and consistency of the global scale atmospheric general circulation.

\subsection{Mean rainfall and circulation features over the SAM region}

We shall now examine the two sets of GCM simulations specifically focusing on the regional features of the SAM. Figure.5 shows the JJAS mean maps of the simulated $850 \mathrm{hPa}$ winds, rainfall and 500 $\mathrm{hPa}$ relative humidity over the Indian subcontinent and adjoining areas. The effect of increased horizontal resolution through zooming is directly evident from improvements in the orographic monsoon precipitation over the narrow mountains of Western Ghats and Myanmar (see Figs.5b, 5e). 
Resolving these narrow mountains is important to anchor the orographic precipitation over the monsoon regions (Xie et al. 2006). Some of the biases in the simulation of monsoon rainfall by the zoom version include underestimation of precipitation over northern Bay of Bengal (BOB) and northeast India and excessive precipitation over south BOB. Also the observed secondary rainfall maximum over the eastern equatorial Indian Ocean is not adequately captured in the model simulation. It is interesting to note that the zoom simulation shows finer details of orographic precipitation anchored along the Himalayan foothills, which are not otherwise properly resolved in the no-zoom case. Another noteworthy aspect is the relative strengthening of the southwesterly monsoon flow over the Arabian Sea, the Indian region and BOB in the zoom version as compared to the no-zoom case. The maximum wind speed in the core of the monsoon low-level jet is found to be $\sim 15 \mathrm{~ms}^{-1}$ for the nozoom case and $\sim 18 \mathrm{~ms}^{-1}$ for the zoom version.

The most striking difference between the two sets of simulations pertains to the circulation and rainfall over the MT region in northern India. The zoom version shows a well-defined cyclonic circulation with westerlies on the southern flanks and easterly winds on the northern flanks of the MT. The cyclonic turning of monsoonal winds over BOB can be clearly noticed in Fig.5a. It is important to note that the monsoon rainfall is well-distributed over the plains of north-central India in the zoom simulation and extends up to northwest India. On the other hand, the spatial extent of the cyclonic circulation in the no-zoom version is limited mostly to the BOB and eastern India, so that the monsoon rainfall is relatively low over the MT zone and does not extend into northwest India. Figures 5(a-b) suggest a close association between the wide-spread rainfall distribution and the cyclonic circulation over the MT in the zoom version. Basically, the advection of moisture from adjoining oceanic areas by the monsoonal winds maintains high humidity levels over the MT region in the zoom version. In fact, it can be noticed that the zoom version shows significantly high relative humidity in mid-tropospheric 
levels over the MT region and the Indian landmass, as compared to the no-zoom simulation (Fig.5c, 5d). The drier conditions over northwest India and MT region in the no-zoom simulation (Fig.5f) are partly due to incursion of dry westerly winds from the sub-tropical desert areas to the west (see Bhat 2006, Krishnamurti et al 2010). In fact, one can notice the intrusion of westerly winds from the IndoPak region into northwest and north India in Fig.5d. Dry air intrusions in the tropical and monsoon regions tend to suppress rainfall through decrease of convective instability and depletion of parcel buoyancy (eg., Brown and Zhang, 1997, Krishnan et al. 2009).

Figure. 6 shows the annual cycle of rainfall and surface temperature averaged over the Indian land region from the model simulations and observations. The peak monsoon rainfall during July and August is well captured in the zoom simulation. Also the summer monsoon rainfall simulation in the zoom version is closer to observations as compared to the no-zoom case particularly during June, July and August (JJA). The root mean square error (RMSE) between the observed and simulated rainfall over India is found to be $1 \mathrm{~mm} \mathrm{day}^{-1}$ for the zoom version and $2 \mathrm{~mm} \mathrm{day}^{-1}$ for the no-zoom case. The underestimation of monsoon rainfall over the South Asian region in the no-zoom simulation is consistently reflected in higher surface air temperatures during JJA in the no-zoom version as compared to the zoom case. It may be noted that the zoom version overestimates the surface air temperatures during October, November and December months. The root mean square error (RMSE) between the observed and simulated surface temperature over India is found to be $1{ }^{\circ} \mathrm{C}$ for the zoom version and $2^{\circ} \mathrm{C}$ for the no-zoom case.

\section{Impacts of high-resolution on moist convective processes}

\subsection{Moist processes over the MT region}

Understanding the moist processes over the MT region is important for gaining insight into the 
distribution of monsoon precipitation in the zoom and no-zoom simulations. Figures.7 (a-c) show maps of precipitable water (ie., vertically integrated specific humidity) for the JJAS season based on ERA reanalysis, the zoom and no-zoom simulations. It can be seen that the precipitable water is considerably underestimated over $\mathrm{BOB}$ and the MT region in the no-zoom simulation. The area-averaged values of precipitable water computed over the MT region $\left(70^{\circ} \mathrm{E}-95^{\circ} \mathrm{E}, 16^{\circ} \mathrm{N}-28^{\circ} \mathrm{N}\right)$ from the ERA reanalysis, the zoom and no-zoom simulations are found to be $54 \mathrm{~kg} \mathrm{~m}^{-2}, 52.5 \mathrm{~kg} \mathrm{~m}^{-2}$ and $47 \mathrm{~kg} \mathrm{~m}^{-2}$ respectively. The zoom simulation captures the precipitable water maxima over BOB, west coast of India. Also it can be noticed that the distribution of precipitable water extends well into the MT region in the zoom simulation. On the other hand, the values of precipitable water over north-central India are much lower in the no-zoom case.

Figures. 7 (d-f) show JJAS mean maps of moist static energy (MSE) vertically integrated from $1000 \mathrm{hPa}$ to $700 \mathrm{hPa}$. Basically, high values of MSE at the surface and lower levels indicate unstable air prone to convective ascent and rainfall (Emanuel, 1994). One can notice high MSE values on the eastern side of the MT and north-eastern India (Fig.7d). The high MSE values on the eastern side of the MT are to some extent captured in the zoom simulation. The MSE values over the MT region are significantly underestimated in the no-zoom simulation. The MSE in the lower troposphere are primarily regulated by specific humidity. The enhanced MSE in the zoom simulation is due to enrichment of water vapor over the BOB, north and northeastern India, while the lower MSE in the nozoom version can arise due to dry air intrusions from the sub-tropics and extratropics (eg., Hastenrath and Lamb, 1977, Bhat 2006, Krishnamurti et al. 2010). The area-averaged values of MSE over the MT region $\left(70^{\circ} \mathrm{E}-95^{\circ} \mathrm{E}, 16^{\circ} \mathrm{N}-28^{\circ} \mathrm{N}\right)$ from the ERA reanalysis, the zoom and no-zoom simulations are found to be $340.6 \mathrm{KJm}^{-2}, 338.8 \mathrm{KJm}^{-2}$ and $334.2 \mathrm{KJm}^{-2}$ respectively. 
The vertical distribution of water vapor over the MT is useful to understand the moist convective processes over the Indo-Gangetic plains during the summer monsoon rainy season. Longitude-pressure cross-sections of specific humidity from the ERA, the zoom and no-zoom simulations are shown in Figs. 8 (a-c). The ERA humidity field shows a zonal gradient with higher humidity to the east and lower humidity to the west of the MT. Notice that high levels of specific humidity (>0.01 kg kg-1) extend vertically almost up to $700 \mathrm{hPa}$ in the eastern side of the MT, whereas they are mostly confined below $900 \mathrm{hPa}$ on the western side (Fig.8a). The east-west gradient of the humidity field along the MT is seen in the zoom and no-zoom simulations. The vertical extent of moisture in the eastern side of the MT is lower in both the simulations as compared to ERA. Nevertheless, it may be noted that the high specific humidity $\left(>0.01 \mathrm{~kg} \mathrm{~kg}^{-1}\right)$ values extend vertically up to $750 \mathrm{hPa}$ in the zoom simulation, but are restricted to lower levels below $850 \mathrm{hPa}$ in the no-zoom case. The troposphere is much drier, even at the lower levels, in the no-zoom case.

The process of heating and moistening of atmosphere through organized cumulus convection is fundamental over the tropics and monsoon environment (Yanai et al. 1973). Tropical meso-scale convective systems (MCS) provide an important link between organized cumulus convection and largescale motion (Houze, 2004). In a recent study, Choudhury and Krishnan (2011) pointed out that latent heating from organized MCS over the MT region can effectively promote the upward development of continental-scale cyclonic circulation well above the mid-tropospheric levels. Figure.8d shows the vertical profiles of relative vorticity $(\zeta)$ averaged over the MT region from ERA (green), the zoom (blue) and no-zoom (purple) simulations. The corresponding plots of the vertical profiles of divergence (D) and vertical velocity $(\omega)$ are shown in Fig.8e and Fig.8f respectively. From Fig.8d, it can be seen that the positive values of $\zeta$ (cyclonic) in the low and middle troposphere are significantly stronger in ERA and the zoom simulation as compared to the no-zoom case. Also note that the cyclonic vorticity 
has deeper vertical extent up to $\sim 450 \mathrm{hPa}$ in both ERA and the zoom simulation, whereas the positive $\zeta$ extends only up to $600 \mathrm{hPa}$ in the no-zoom case. The anticyclonic (negative $\zeta$ ) vorticity in the uppertroposphere, with maximum around $150 \mathrm{hPa}$, is associated with the Tibetan high. It can be noted that the upper-level anticyclonic vorticity is stronger in ERA and zoom simulation as compared to the nozoom case.

The vertical profiles of divergence (D) show stronger convergence (negative) from $1000 \mathrm{hPa}$ to $700 \mathrm{hPa}$ in ERA and the zoom simulation as compared to the no-zoom case. In the no-zoom case, the vertical extent of convergence is shallow and restricted to lower levels below $850 \mathrm{hPa}$. Note that the upper-level divergence is relatively stronger in ERA and the zoom simulation as compared to the nozoom case. As compared to ERA, the maximum vertical velocity is overestimated in the zoom simulation and shows differences in the placement of the level of maximum vertical velocity. On the other hand, the magnitude of upward velocity is much smaller in the no-zoom simulation. It is important to notice the steady build up of upward motions (negative $\omega$ ) in ERA and the zoom simulation in Fig.8e. Basically, the stronger convergence at the lower and mid tropospheric levels enhances vorticity stretching leading to generation of cyclonic vorticity over the MT region in the zoom simulation (see Choudhury and Krishnan, 2011).

\subsection{Simulation of rainfall and circulation during active monsoon conditions}

On sub-seasonal time-scales, the Indian summer monsoon is characterized by active and break spells in the monsoon rainfall activity. Active monsoons are characterized by enhanced precipitation over central India and the MT region arising from interactions between the moist convective processes and the southwesterly monsoon circulation (eg. Rajeevan et al. 2010, Choudhury and Krishnan 2011). We now focus on the simulation of the active monsoon conditions in the zoom and no-zoom 
configurations. Figure 9a shows composite map of observed precipitation based on active monsoon days as defined by Rajeevan et al. (2010). Using the daily gridded observed rainfall dataset from the India Meteorological Department (IMD), the composite map was created by averaging over all the active monsoon days. Rajeevan et al. (2010) identified active monsoon phases as episodes when the normalized rainfall anomaly over a core monsoon zone in north-central India exceeds one standard deviation for at least three consecutive days. We have adopted the same method for determining the active monsoon phases in the GCM simulations. It is noted that the total number of active monsoon cases in the 10-member zoom and no-zoom simulations were 14 and 11 respectively. The precipitation composites, based on active monsoon days, for the zoom and no-zoom simulations are shown in Fig.9b and Fig.9c respectively. The observed rainfall composite shows a well-defined east-west band of maximum precipitation over central and northern India, together with enhanced precipitation over the west coast and northeast India (Fig.9a). The zoom simulation of the active monsoon condition shows enhanced precipitation over the west coast and a wide region of central and northern India. It is realized that the zoom simulation has certain biases such as the low rainfall over western India and the head Bay of Bengal. However, the important point is that rainfall along the MT zone is considerably better resolved in the zoom version (Fig.9b) as compared to the no-zoom case (Fig.9c). In fact, it may be noted that the latter seriously underestimates the rainfall along the MT axis.

Composite maps of the $850 \mathrm{hPa}$ winds during the active phases based on the ERA, zoom and no-zoom runs are shown in Figs.9 (d-f) respectively. The ERA $850 \mathrm{hPa}$ wind composite shows a welldefined east-west oriented cyclonic circulation extending from northwest India up to the head Bay of Bengal. This feature is accompanied by a monsoon cross-equatorial flow with strong southwesterly winds to the north-of-equator. The cyclonic circulation around the MT and the strong southwesterly monsoon winds are captured in the zoom simulation, although the axis of the cyclonic circulation has a 
slightly different orientation in the southeast-northwest direction (Fig.9e). In the no-zoom version, the horizontal scale of the cyclonic circulation is mostly limited to eastern and central India and the southwesterly winds over the Arabian Sea and Indian region are not as strong as in the zoom version.

During active monsoons the mid-troposphere is characterized by a continental scale cyclonic vortex centered over the South Asian MT region which extends westward up to the African monsoon region (Choudhury and Krishnan, 2011). This feature is clearly evident in the $500 \mathrm{hPa}$ winds composite of active monsoons from the ERA dataset (Fig.10a). Notice that the cyclonic vortex around the MT is centered around $20^{\circ} \mathrm{N}$ with easterly winds extending up to $28^{\circ} \mathrm{N}$. The subtropical westerlies are located mostly to the north of $35^{\circ} \mathrm{N}$ and the anticyclonic circulation over the Arabian Desert indicates subsidence over the region. The zoom simulation captures the continental scale cyclonic pattern as well as the cyclonic circulation over the MT region with easterlies extending up to $29^{\circ} \mathrm{N}$ on the northern flanks. In conjunction with the strong meridional expanse of the mid-level cyclonic vortex, one can notice two distinct sub-tropical anticyclones in ERA and the zoom simulation over the (a) Arabian Desert (b) Southwest China $\left(\sim 100^{\circ} \mathrm{E}, 30^{\circ} \mathrm{N}\right)$ (see Figs.10a-b). The meridional extent of the cyclonic vortex over the MT zone is relatively smaller in the no-zoom simulation, as compared to the zoom version, with the easterlies on its northern flanks typically extending up to $26^{\circ} \mathrm{N}$ (see Fig. 10c). Also, it may be noted that the cyclonic vortex is positioned relatively southward with a local maximum around $\left(80^{\circ} \mathrm{E}, 18^{\circ} \mathrm{N}\right)$. It is interesting to note that the restricted meridional extent of the MT mid-level cyclonic vortex in the no-zoom simulation is accompanied by an anticylonic ridge with its axis located near the $30^{\circ} \mathrm{N}$ latitude (Fig.10c).

A comparison of active monsoon composites of the $200 \mathrm{hPa}$ circulation for the ERA, the zoom and no-zoom simulations is presented in Figs. 10(d-f). The large-scale structure of the upper- 
tropospheric Tibetan anticyclone, characterized by a prominent longitudinal elongation, can be seen in the ERA and zoom simulation. Hsu and Plumb (2000) pointed out that an elongated Tibetan anticyclone can become unstable and periodically shed eddies on the westward side. The Tibetan anticyclone in the no-zoom simulation is more pronounced regionally between $60^{\circ} \mathrm{E}-110^{\circ} \mathrm{E}$ and the westward elongation is not as prominent as in the no-zoom version (Fig.10f). The cross-equatorial upper-level winds diverging from the Tibetan anticyclone and the tropical easterly jet are weaker in magnitude in the no-zoom simulation (Fig.10f) as compared to the ERA and zoom simulation (Figs.10d-e).

\subsection{Simulation of monsoon lows and depressions with and without telescopic zooming}

Monsoon low pressure systems (LPS) which comprise of lows, depressions and deep-depressions are important rain producing synoptic disturbances over the Indian region during the summer monsoon season. These disturbances generally form in the Bay of Bengal and move in a west-northwest direction along the quasi-stationary monsoon trough across north-central India (eg., Koteswaram and Rao, 1963, Rao, 1976, Sikka 2006). Studies have shown that the existence of combined barotropicbaroclinic instability of the mean monsoon flow is a necessary condition for the generation of monsoon disturbances (e.g., Keshavamurty et al., 1978, Goswami et al., 1980, Mishra and Salvekar et al, 1980, Satyan et al., 1980, Dash and Keshavamurty, 1982). On the other hand, the energetics of monsoon depressions appears to be primarily maintained by cumulus convection and moist processes (see Krishnamurti et al., 1976). Thus, it would be of interest to investigate the simulation of monsoon LPS in the zoom and no-zoom experiments from the perspective of understanding the moist convective processes over the MT region.

We have identified monsoon LPS using the daily sea level pressure (SLP) and wind fields from 
the zoom and no-zoom simulations following the procedure similar to Lal et al. (1995). The criterion for identifying the vortex centers along the LPS tracks is based on specified thresholds of $850 \mathrm{hPa}$ relative vorticity, SLP, and surface wind speed. Accordingly, a LPS vortex is identified when (a) Relative vorticity at $850 \mathrm{hPa}$ exceeds $2.0 \times 10^{-5} \mathrm{~s}^{-1}$ (b) Wind speed at $1000 \mathrm{hPa}$ exceeds $15 \mathrm{~ms}^{-1}$ and $\mathrm{SLP}<998 \mathrm{hPa}$ within a $3^{\circ}$ x $3^{\circ}$ grid domain (c) Events with minimum duration of 3 days are only considered (d) The co-ordinates of the minimum SLP correspond to the centre of the LPS. The tracks of monsoon LPS based on the zoom and no-zoom simulations are shown in Fig.11a and Fig.11b respectively. In both cases, one can notice west-northwestward tracks of the monsoon LPS. However, the tracks in the zoom simulation extend farther westward into northwest India as compared to the nozoom simulation. Also, it is interesting to note that the mean track in the zoom simulation is located more northward relative to that in the no-zoom simulation. Using the track data, we also computed the LPS density on $1^{\circ} \times 1^{\circ}$ grid boxes by counting the number of LPS passing through any particular grid box. Maps of LPS density for the zoom and no-zoom simulation are shown in Fig. 11c and Fig.11d respectively. It can be seen that the LPS density magnitudes are significantly higher in the zoom simulation as compared to the no-zoom run. It is also important to note that the LPS density values in the zoom simulation extend more westward and northward as compared to the no-zoom case.

Figures.12 (a-b) illustrate the $850 \mathrm{hPa}$ streamlines and rainfall associated with a typical monsoon LPS in the zoom and no-zoom simulations respectively. The streamlines and rainfall are averaged over the entire period of the LPS. It can be noticed that the cyclonic circulation in the zoom simulation is elongated more westward and extends farther west into northwest India and Pakistan. On the other hand, the monsoon LPS cyclonic circulation in the no-zoom simulation is mostly limited to central and eastern India. Also it may be noted that the east-west axis of the cyclonic circulation is located more northward in the zoom simulation as compared to the no-zoom case. In the zoom 
experiment, the simulated rainfall during the monsoon LPS covers a large area of central-north India along the MT zone and is oriented along the southern side of the cyclonic circulation. Figure.12a also shows significant rainfall along the Western Ghats in association with the strong monsoon westerly winds. In the no-zoom simulation (Fig.12b), the rainfall band associated with the monsoon LPS is located considerably southward as compared to the zoom version. Interestingly, the no-zoom simulation shows negligible rainfall over north-central India and the Indo-Gangetic plains, whereas enhanced precipitation can be seen all along the Himalayan foothills in association with a cyclonic circulation in the sub-tropical westerly winds (Fig.12b). Such a condition of decreased monsoon rainfall/convection over the plains of north-central India and enhanced precipitation/convection over the Himalayan foothills is generally observed during "breaks" in the Indian summer monsoon (e.g., Ramamurthy, 1969, Krishnan et al., 2000, 2009, Gadgil et al. 2003, Rajeevan et al. 2010).

Based on the discussions above, it can be inferred that the presence of adequate moisture in the low and mid-tropospheric levels over the MT zone is important for providing a favorable environment for the transient monsoon synoptic disturbances to develop and extend well into northwest India. Essentially, the ability of the zoom simulation to confine moisture through the continental scale cyclonic circulation encourages the organization of moist convective processes over the MT zone. On the other hand, inadequate moisture in the no-zoom simulation leads to suppression of moist convective processes particularly towards the western side of the MT zone. We shall return to this point later while discussing the overall results in the last section.

\subsection{Organization of monsoon meso-scale convective systems}

Organization of tropical convection involves interactions between the cumulus scale and large-scale circulation which are mediated through the tropical MCS (e.g., Krishnamurti et al. 1976, Mapes and 
Houze, 1995, Houze, 2004). The organization of MCS over the MT region is evident during active monsoon conditions which are often accompanied by enhanced activity of monsoon LPS (see Krishnan et al., 2011, Choudhury and Krishnan, 2011). An important element observed during active monsoons is the pre-dominance of moderate-to-heavy rainfall over the plains of central and north India (eg., Joshi and Rajeevan, 2006, Rajeevan et al. 2010). In this section, we shall focus on understanding the relationship between the MCS activity over the MT region and the large-scale summer monsoon circulation in the zoom and no-zoom simulations.

Using outputs of daily rainfall from the model simulations, we have employed an objective procedure to quantify the organization of MCS activity over the MT zone based on counting the frequency of moderate-to-heavy rainfall cases covering the domain $\left(70^{\circ} \mathrm{E}-90^{\circ} \mathrm{E}, 16^{\circ} \mathrm{N}-28^{\circ} \mathrm{N}\right)$. An outline of the objective procedure is presented below:

(a) With 10 realizations of the model each covering the June to September (120 days $)^{2}$ monsoon rainy season, we have a total of $1200(=120 \times 10)$ rainfall values at each grid-point. This allows us to construct a rainfall time-series $(n=1200)$ at each grid-point by sequentially arranging the 10 model realizations. In this time-series, the data points $(1,2,3 \ldots 120)$ are from the first realization; the data points $(121,124 \ldots 240)$ correspond to the second realization; ... the points $(1081,1900, \ldots 1200)$ correspond to the tenth realization.

(b) In the next step, we determine the thresholds for moderate and heavy rainfall events at every grid-point based on the IMD criterion. According to this criterion, the $75^{\text {th }}$ percentile is the threshold for moderate rainfall and the $95^{\text {th }}$ percentile is the threshold for heavy rainfall (Joshi and Rajeevan, 2006). 
(c) Knowing the moderate and heavy rainfall thresholds, we then determine if the rainfall on a particular day at a given grid-point lies between the two thresholds. This procedure is applied at all the grid-points. By this process, we obtain the total count of moderate-to-heavy rainfall cases in the entire MT domain on any particular day. A higher count of moderate-to-heavy rainfall on any given day implies large-scale organization of the MCS at that point of time; whereas a lower count is indicative of localized convective activity.

By following the above steps, one can generate the daily time-series $(n=1200)$ of the frequency count of moderate-to-heavy rainfall over the MT domain $\left(70^{\circ} \mathrm{E}-90^{\circ} \mathrm{E}, 16^{\circ} \mathrm{N}-28^{\circ} \mathrm{N}\right)$. It may be noted that the zoom and no-zoom versions have 1500 and 273 grid-points respectively over the MT domain. Thus, the unit of frequency in the zoom version is number of counts per $\mathrm{N}_{\mathrm{z}}(=1500)$; and number of counts per $\mathrm{N}_{\mathrm{nz}}(=273)$. Figures.13 (a-b) show the time-series of the frequency count (FC) of moderateto-heavy rainfall over the MT domain for the zoom and no-zoom simulations. For the zoom experiment, the mean and standard-deviation of the FC time-series are found to be 203 per 1500 ( $0.14)$ and 183 per $1500(\sim 0.12)$ respectively. The corresponding values for the no-zoom experiment are found to be 10 per $273(\sim 0.04)$ and 13 per $273(\sim 0.05)$ respectively. In other words, the mean frequency of moderate-to-heavy rainfall cases is about $14 \%$ with respect to (w.r.t) the total grids in the MT domain for the zoom experiment; whereas the mean frequency is about $4 \%$ of the total grids over the same domain for the no-zoom experiment.

In order to examine the relationship between the large-scale monsoon circulation and the organization of MCS over the MT zone, we regress the model simulated horizontal wind field at 850 hPa upon the time-series of frequency count of moderate-to-heavy rainfall (Fig.13). Before performing the regression analysis, the daily horizontal winds from the 10 model realizations were first arranged 
sequentially just as in the case of the rainfall time-series. The patterns generated by regressing the 850 $\mathrm{hPa}$ winds on the index of frequency count of moderate-to-heavy rainfall are shown in Fig.14a and Fig.14b for the zoom and no-zoom simulations respectively. In the zoom experiment, the regression pattern shows a continental scale cyclonic vortex around the MT zone. A prominent westerly pattern can be seen extending from the Horn of Africa across the Arabian Sea into the Indian landmass and the Bay of Bengal in Fig.14a. It is also important to note that the westerly pattern in the zoom case has a wide meridional extent from $\sim 8^{\circ} \mathrm{N}$ to $20^{\circ} \mathrm{N}$ covering much of the west coast of India. Likewise the pattern of easterlies on the northern flanks of the cyclonic vortex is quite strong in the zoom simulation. In contrast, the no-zoom simulation shows a much weaker pattern of westerlies over the Arabian Sea and Indian region. Further, it can be noted that the meridional extent of the westerly pattern and the scale of the cyclonic circulation is much smaller, while the easterly pattern to the north is considerably weak in Fig.14b. The above results suggest that the scale interaction between the organization of MCS over the MT region and the large-scale monsoonal winds is rather strong and robust in the zoom experiment, but much weaker in the no-zoom version.

\section{Discussions and conclusions}

The present work has addressed some important scientific questions concerning scale interactions in the SAM region using the LMDZ global stretched-grid GCM with a $35-\mathrm{km}$ telescopic zooming over South and West Asia. The motivation for this study stems from the fact that interactions among multiple scales (i.e., large, synoptic, meso and cumulus scales) are central to many key elements of the SAM system - viz., the space-time distribution of rainfall, the large-scale organization of moist convective processes over the MT zone, the evolution of transient monsoon LPS etc. Given the inherent limitations of coarse resolution GCMs (grid size 200-300 km) in capturing smaller scale processes like the monsoon MCS, it is desirable to understand if a global GCM with high-resolution 
zooming over the SAM region would be a feasible framework to address this issue.

Based on the above premise, we have conducted two sets of 10-member ensemble simulations of the LMDZ GCM with and without telescopic zooming over the SAM region, and validated the simulations with observed and reanalysis datasets. In addition to preserving the realism and consistency of the global general circulation features, it is interesting to note that the zoom simulation exhibits remarkable improvements in capturing the regional monsoon rainfall and circulation over South Asia. The monsoon precipitation over central-north India, the Indo-Gangetic plains and the rainfall maxima along the narrow Western Ghats and the mountain slopes of Northeast India and Myanmar are far more realistically simulated in the zoom version as compared to the no-zoom counterpart. Furthermore, the zoom simulation out-performs the no-zoom version in capturing the cyclonic circulation and the associated humidity and moist-static energy fields around the MT zone, together with more realistic vertical profiles of relative vorticity, divergence and vertical velocity over the region. Likewise the zoom simulation also provides a better portrayal of the active monsoon conditions of regional rainfall and circulation, the west-northwest tracks of monsoon LPS that emanate from the Bay of Bengal region, and the distribution of moderate-to-heavy rainfall events due to organized activity of MCS over the MT zone. By consolidating these results, it can be summarized that the zoom simulation not only enhances the regional details of the SAM precipitation, but also provides greater value addition through improved representation of the monsoonal scale interactions and moist convective processes.

The present findings suggest that the improved representation of moist convective processes in the zoom simulation involves the formation of a continental scale cyclonic circulation around the MT zone. This cyclonic circulation extends well above $500 \mathrm{hPa}$ and maintains a moist environment with high moist static energy that is conducive for the organization of convective processes over the MT 
region. On the other hand, the cyclonic circulation in the no-zoom simulation is confined mostly to the eastern part of the MT zone, with drier conditions prevailing over the western and central parts of the MT due to entrainment of dry air from the west in the mid-tropospheric levels across the Indo-Pak area. Dry air intrusions in the mid-tropospheric levels tend to inhibit convective instability and suppress convection (eg., Bhat, 2006, Krishnan et al. 2009, Krishnamurti et al. 2010) and discourage the growth of deep convective clouds by depleting parcel buoyancy (Brown and Zhang, 1997).

From the present results, it is noted that the drying of the lower and mid-tropospheric levels in the no-zoom simulations suppresses the organization of MCS over the MT zone and restricts the westward extent of the monsoon LPS. In the case of the zoom simulation, the organization of MCS over the MT zone tends to be favored through confinement of moisture by interactive feedbacks between the large-scale monsoon flow, the continental scale cyclonic vortex and the re-circulating monsoon LPS that traverse westward along the axis of the MT. Recent studies have pointed out that vortices in the tropical easterly waves over the Atlantic and eastern Pacific can develop into tropical depressions through wave-vortex interaction in a manner similar to the development of a marsupial infant in its mother's pouch (eg., Dunkerton et.al. 2009, Wang et al. 2012). Such a wave-vortex interaction is favored under conditions of weak vortex deformation and moisture containment provided the parent wave is well maintained, so that the above environmental conditions can encourage the aggregation of mesoscale vortices to produce convective heating (Dunkerton et al. 2009). It is conceivable that similar interactions might occur during the evolution and growth of monsoon LPS due to feedbacks among the large-scale monsoon flow, the deep continental scale vortex and the recirculating LPS vortices. In fact, it has been highlighted that the latent heating distribution from organized MCS exerts dominant influence on the intensity and vertical extent of the continental-scale cyclonic circulation around the MT zone (see Choudhury and Krishnan, 2011). 
While it is realized that the moist convective processes in a GCM are sensitive to the treatment of physics and cumulus parameterization schemes, our understanding suggests that enhancing the resolution of GCMs would be crucial for accurately representing the moisture gradients over northwest India and Indo-Pak region in the lower and mid-tropospheric levels. Boos and Kuang (2010) and Nie et al. (2010) have hypothesized that resolving the narrow orography of the Himalayas and the adjacent mountain ranges is important for sustaining strong monsoons by insulating the warm and moist air (ie., high entropy air) over the Indian landmass from the cold and dry extra-tropics (low entropy air). Model sensitivity experiments indicate that the Hindu-Kush mountains can also affect the strength of the Indo-Pak low during the summer monsoon season (Bollasina and Nigam, 2010). It is important to recognize that the western part of the MT is a border area that separates a highly moist environment on the eastern side from the highly arid locations to the west. Therefore, the use of high-resolution models is essential to accurately resolve the moisture gradients over northwest India, Indo-Pak region and the Hindu-Kush mountains, which in turn allows proper representation of the moist convective processes over the MT region. Finally, the overall synthesis from this work enhances our confidence in acknowledging the prospects to improve the quality of monsoon rainfall simulations and forecasts over the South Asian region through the use of stretched-grid global GCMs with fine-scale resolution over the monsoon region.

\section{Acknowledgments}

RK and TPS thank Prof. B.N. Goswami, the Director, Indian Institute of Tropical Meteorology (IITM) for extending all support for this research work. IITM is fully funded by the Ministry of Earth Sciences, Government of India. 


\section{References}

Adler RF, et al. (2003) The version-2 Global Precipitation Climatology Project (GPCP) monthly precipitation analysis (1979-Present). J Hydrometeor 4: 1147-1167

Alexander G, Keshavamurty RN, De US, Chellappa R, Das SK, Pillai PV (1978) Fluctuations of monsoon activity. Indian J Meteor Geophys 29: 76-87

Bhaskaran B, Jones RG, Murphy JM, Noguer M (1996) Simulations of the Indian summer monsoon using a nested climate model: Domain size experiments. Clim Dyn 12: 573-587

Bhat GS (2006) The Indian drought of 2002 - A sub-seasonal phenomenon?. Quart J Roy Meteor Soc 132: $2583-2602$

Bollasina M, Nigam S (2010) The summertime "Heat" low over Pakistan / Northwestern India: Evolution and Origin. Clim Dyn, doi: 10.1007/s00382-010-0879-y

Boos WR, Kuang Z (2010) Dominant control of the South Asian monsoon by orographic insulation versus plateau heating. Nature 463: doi:10.1038/nature08707

Brown RG, Zhang C (1997) Variability of mid tropospheric moisture and its effect on cloud-top height distribution during TOGA COARE. J Atmos Sci 54: 2760-2774

Choudhury AD, Krishnan R (2011) Dynamical response of the South Asian monsoon trough to latent heating from stratiform and convective precipitation. J Atmos Sci 68:1347-1363

Das PK (1986) Monsoons. WMO Rep 613, 115 pp.

Dash SK, Keshavamurty RN (1982) Stability of mean monsoon zonal flow Beitr Phys Atmosph 55: 299-310

Dash SK, Shekhar MS, Singh GP (2006) Simulation of Indian summer monsoon circulation and rainfall using RegCM3. Theor Appl Climatol 86: 161-172

Ding Q, Wang B (2007) Intraseasonal teleconnection between the Eurasian wavetrain and Indian 
summer monsoon. J Clim 20: 3751-3767

Dunkerton TJ, Montgomery MT, Wang Z (2009) Tropical cyclogenesis in a tropical wave critical layer: Easterly waves. Atmos Chem Phys 9: 5587-5646

Emanuel KA (1993) A cumulus representation based on the episodic mixing model: the importance of mixing and microphysics in predicting humidity. A M S Meteorol Monographs 24: 185-192

Emanuel KA, Neelin JD, Bretherton CS (1994) On large-scale circulations in convective atmospheres, Q J R Meteorol Soc 120: 1111-1143

Enomoto TB, Hoskins J, Matsuda Y (2003) The formation mechanism of the Bonin high in August. QJ R Meteorol Soc 587: 157-178

Fox-Rabinovitz MS, Cote J, Deque M, Dugas B, McGregor J (2006) Variable-Resolution GCMs: Stretched-Grid Model Intercomparison Project (SGMIP). J Geophys Res 111: D16104, doi:10.1029/2005JD006520.

Gadgil S (2003) The Indian monsoon and its variability. Annu Rev Earth Planet Sci 31: 429-467

Gadgil S, Sajani S (1998) Monsoon precipitation in the AMIP runs. Clim Dyn 14: 659-689

Goswami BN, Ajayamohan RS, Xavier PK, Sengupta D (2003) Clustering of synoptic activity by Indian summer monsoon intraseasonal oscillations. Geophys Res Lett 30: 1431, doi:10.1029/2002GL016734

Goswami BN, Keshavamurthy RN, Satyan V (1980) Role of barotropic-baroclinic instability for the growth of monsoon depressions and mid-tropospheric cyclones. Proc Ind Acad Sci 89: 79-97

Hastenrath S, Lamb P (1977) Climatic Atlas of the Tropical Atlantic and Eastern Pacific Oceans. University of Wisconsin Press: Madison, $112 \mathrm{pp.}$

Hourdin F, Ionela M, Bony S, Francis Codron, Jean-Louis Dufresne, Laurent Fairhead, Marie-Ange, le Filiberti, Friedlingstein P, Grandpeix JY, Krinner G, Phu LeVan, Zhao-Xin Li, LottHouze F (2006) The LMDZ4 general circulation model: climate performance and sensitivity to 
parametrized physics with emphasis on tropical convection. Clim Dyn 27: 787-813, DOI $10.1007 / \mathrm{s} 00382-006-0158-0$

Houze RA (2004) Mesoscale convective systems. Rev Geophys 42: 10.1029/2004RG000150,43 pp

Hsu CJ, Plumb RA (2000) Non-axisymmetric thermally driven circulations and upper tropospheric monsoonal dynamics. J Atmos Sci 57: 1254-1276

Jacob D, Podzum R (1997) Sensitivity studies with the regional climate model REMO. Meteor Atmos Phys 63: 119-129

Joseph PV, Sabin TP (2008) An ocean-atmosphere interaction mechanism for the active break cycle of the Asian summer monsoon. Clim Dyn 30: 553-566, DOI: 10.1007/s00382-007-0305-2

Joshi U, Rajeevan M (2006) Trends in precipitation extremes over India. Tech. Rep. 3, National Climate Centre, $25 \mathrm{pp}$.

Keshavamurty RN, Asnani GC, Pillai PV, Das SK (1978) Some studies on the growth of monsoon disturbances. Proc Indian Acad Sci 87: 61-75

Keshavamurty RN, Awade ST (1974) Dynamical abnormalities associated with drought in the Asiatic summer monsoon. Indian J Meteor Geophys 25: 257-266

Kitoh A, Kusunoki S (2009) East Asian summer monsoon simulation by a 20-km mesh AGCM. Clim Dyn, doi:10.1007/s00382- 007-0285-2

Koteswaram P (1958) The easterly jet stream in the tropics. Tellus 10: 43-56

Koteswaram P, Rao NSB (1963) The structure of the Asian summer monsoon. Aust Meteor Mag 42: 35-36

Krishnamurti TN (1971) Tropical east-west circulations during the northern summer. J Atmos Sci 28: 1342-1347

Krishnamurti TN (1973) Tibetan high and upper tropospheric tropical circulation during northern summer. Bull Amer Meteor Soc 54: 1234-1249 
Krishnamurti TN, Bhalme HN (1976) Oscillations of a monsoon system. Part I: Observational aspects. J Atmos Sci 33: 1937-1954

Krishnamurti TN, Kanamitsu M, Godbole RV, Chang CB, Carr F, Chow JH (1976) Study of a monsoon depression II. Dynamical structure. J Meteor Soc Japan 54: 208-225

Krishnamurti TN, Surgi N (1987) Observational aspects of the summer monsoon. Monsoon Meteorology, C.-P. Chang and T.N. Krishnamurti, Eds., Oxford University Press, 3-25.

Krishnamurti TN, Thomas A, Simon A, Vinay Kumar (2010) Desert air incursions, an overlooked aspect, for the dry spells of the Indian summer monsoon. J Atmos Sci 67: 3423-3441

Krishnan R, Sabin TP, Ayantika DC, Kitoh A, Sugi M, Murakami H, Turner AG, Slingo JM, Rajendran K (2012) Will the South Asian monsoon overturning circulation stabilize any further?. Clim Dyn, DOI 10.1007/s00382-012-1317-0

Krishnan R, Ayantika DC, Kumar V, Pokhrel S (2011) The long-lived monsoon depressions of 2006 and their linkage with the Indian Ocean Dipole. Int J Climatol, doi:10.1002/joc.2156

Krishnan R, Vinay K, Sugi M, Yoshimura J (2009) Internal feedbacks from monsoon-midlatitude interactions during droughts in the Indian summer monsoon. J Atmos Sci 66: 553-578

Krishnan R, Zhang C, Sugi M (2000) Dynamics of breaks in the Indian summer monsoon. J Atmos Sci 57:1354-1372

Lal M, Bengtsson L, Cubasch U, Esch M, Schlese U (1995) Synoptic scale disturbances of the Indian summer monsoon as simulated in a high resolution climate model. Climate Research 5:243-258

Lee DK., Suh MS (2000) Ten-year East Asian summer monsoon simulation using a regional climate model (RegCM2). J Geophys Res 105: 29565-29577

Mapes B, Houze R Jr, (1995) Diabatic divergence profiles in western Pacific mesoscale convective systems. J Atmos Sci 52: 1807-1828

McGregor JL (1996) Semi-Lagrangian advection on conformal cubic grids. Mon Wea Rev 124: 1311- 
Mishra SK, Salvekar PS (1980) Role of baroclinic instability in the development of monsoon disturbances. J Atmos Sci 37: 383-394

Nie Ji, William R Boos, Kuang Z (2010) Observational evaluation of a convective quasi-equilibrium view of monsoon. $J$ clim 23: 4416-4428

Raghavan K, (1973) Tibetan anticyclone and tropical easterly jet. Pure and Applied Geophysics 110: 2130-2142, DOI: 10.1007/BF00876576

Rajeevan M, Bhate J, Kale JD, Lal B (2006) High resolution daily gridded rainfall data for the Indian region: analysis of break and active monsoon spells. Curr Sci 91: 296-306

Rajeevan M, Gadgil S, Bhate J (2010) Active and break spells of the Indian summer monsoon. Proc Indian Acad Sci 119: 229-247

Rajendran K, Kitoh A (2008) Indian summer monsoon in future climate projection by a super highresolution global model. Curr Sci 95:1560-1569

Rajendran K, Kitoh A, Srinivasan J, Mizuta R, Krishnan R (2012) Monsoon circulation interaction with Western Ghats orography under changing climate- Projection by a 20-km mesh AGCM. Theoretical and Applied Climatology, DOI: 10.1007/s00704-012-0690-2.

Ramamurthy K (1969) Monsoon of India: Some aspects of the 'break' in the Indian southwest monsoon during July and August. Forecasting Manual IV-18.3, India Meteorological Dept, 157

Raman CRV, Rao YP (1981) Blocking highs over Asia and monsoon droughts over India. Nature 289: $271-273$

Ramaswamy C (1962) Breaks in the Indian summer monsoon as a phenomenon of interaction between the easterly and the subtropical westerly jet streams. Tellus 14A: 337-349

Rao YP (1976) Southwest monsoon India Meteorological Department. Meteorological Monograph 
Synoptic Meteorology, No.1/1976, Delhi, 367 pp.

Rayner NA, Parker DE, Horton EB, Folland CK, Alexander LV, Rowell DP, Kent EC, Kaplan A (2003)

Global analyses of sea surface temperature, sea ice, and night marine air temperature since the late nineteenth century. J Geophys Res 108:D144407. doi:10.1029/2002JD002670

Rodwell MJ, Hoskins BJ (1996) Monsoons and the dynamics of deserts. Q J R Meteorol Soc 122: 1385-1404. doi: 10.1002/qj.49712253408

Satyan V, Keshavamurty RN, Goswami BN, Dash SK, Sinha HSS (1980) Monsoon cyclogenesis and large scale flow patterns over South Asia. Proc Indian Acad Sci 89: 277-292

Sikka DR (2006) A study on the monsoon low pressure systems over the Indian region and their relationship with drought and excess monsoon seasonal rainfall. COLA Technical Report CTR217.

Simmons AS, Uppala D Dee, Kobayashi S (2006) ERAInterim: New ECMWF reanalysis products from 1989 onwards. ECMWF Newsletter 110, ECMWF, Reading, United Kingdom, 25-35. Available online at http://www.ecmwf.int/publications/newsletters/pdf/110_rev.pdf.

Vernekar AD, Ji Y (1999) Simulation of the onset and intraseasonal variability of two contrasting summer Monsoons. J Clim 12: 1707-1725

Wang Z, Montgomery MT, Fritz C (2012) A first look at the structure of the wave pouch during the 2009 PREDICT-GRIP dry runs over the Atlantic. Mon Wea Rev 140: 1144-1163, doi: 10.1175/MWR-D-10-05063.1

Xie SP, Xu H, Saji NH, Wang Y (2006) Role of narrow mountains in large-scale organization of Asian monsoon convection. J Clim 19:3420-3429

Yanai M, Esbensen S, Chu J (1973) Determination of bulk properties of tropical cloud clusters from large-scale heat and moisture budget. J Atmos Sci 30: 611-627

Zhou T, Li Z (2002) Simulation of the East Asian summer monsoon using a variable resolution 
atmospheric GCM. Clim Dyn 19: 167-180

\section{Figure Captions}

Figure 1. (a) Model grids for entire global domain (shaded area denotes grid-size $\leq 35 \mathrm{~km}$ ) (b) Topography (m) and model grids over the Asian region.

Figure 2. Spatial maps of seasonal rainfall $\left(\mathrm{mm} \mathrm{day}^{-1}\right)$ for the June-July-August-September (JJAS) from a) ERA Interim b) Zoom and c) No-zoom simulation.

Figure 3. Spatial distributions of JJAS mean $850 \mathrm{hPa}$ winds $\left(\mathrm{ms}^{-1}\right)$ a) ERA Interim b) Zoom and c) No-zoom simulation respectively d) Latitudinal variation of the zonally averaged zonal winds $\left(\mathrm{ms}^{-1}\right)$ at $850 \mathrm{hPa}$.

Figure 4. Spatial distributions of JJAS mean $200 \mathrm{hPa}$ winds $\left(\mathrm{ms}^{-1}\right)$ a) ERA Interim b) Zoom and c) No-zoom simulation respectively d) Latitudinal variation of the zonally averaged zonal winds $\left(\mathrm{ms}^{-1}\right)$ at $200 \mathrm{hPa}$.

Figure 5. JJAS mean fields from zoom (left) and no-zoom (right) simulations (a, d) winds at $850 \mathrm{hPa}$ $\left(\mathrm{m} \mathrm{s}^{-1}\right)(\mathrm{b}, \mathrm{e})$ mean precipitation $\left(\mathrm{mm} \mathrm{day}^{-1}\right)$ and $(\mathrm{c}, \mathrm{f})$ relative humidity at $500 \mathrm{hPa}$.

Figure 6. Climatological annual cycles of rainfall $\left(\mathrm{mm} \mathrm{day}^{-1}\right)$ and surface temperature $\left({ }^{\circ} \mathrm{C}\right)$ (line) over the Indian landmass from the zoom and no-zoom simulations. The observed temperature is based on the CRU dataset and precipitation is based on the IMD dataset.

Figure 7. Spatial map of total precipitable water $\left(\mathrm{kg} \mathrm{m}^{-2}\right)$ for JJAS season (left column). Moist static

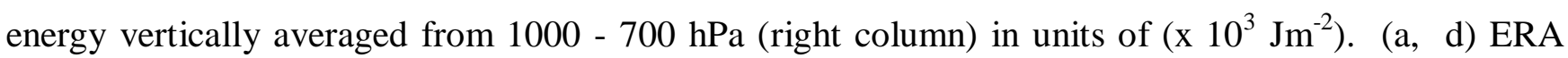
Interim (b, e) Zoom (c, e) No-zoom simulation.

Figure 8. Longitude - Pressure cross-section of specific humidity $\left(\mathrm{kg} \mathrm{kg}^{-1}\right)$ averaged over the MT zone $\left(16^{\circ} \mathrm{N}-28^{\circ} \mathrm{N}\right)$ (a) ERA Interim (b) Zoom simulation (c) No Zoom simulation. Vertical profiles 
averaged over the monsoon trough region $\left(16^{\circ} \mathrm{N}-28^{\circ} \mathrm{N}, 65^{\circ} \mathrm{E}-100^{\circ} \mathrm{E}\right)$ (d) Relative vorticity $\left(\mathrm{x} 10^{5} \mathrm{~s}^{-1}\right)$ (e) divergence $\left(\times 10^{5} \mathrm{~s}^{-1}\right)$ (f) vertical velocity $\left(\mathrm{hPa} \mathrm{s}^{-1}\right)$. The profiles for ERA Interim, Zoom and Nozoom simulations are shown in green, blue and purple lines respectively.

Figure 9. Spatial map of rainfall $\left(\mathrm{mm} \mathrm{day}^{-1}\right)$ based on active monsoon days a) Observed IMD gridded $(1 \mathrm{deg}$ x $1 \mathrm{deg})$ rainfall dataset b) Zoom simulation c) No-zoom simulation. Winds $\left(\mathrm{ms}^{-1}\right)$ at $850 \mathrm{hPa}$ d) ERA Interim e) Zoom simulation f) No-zoom simulation.

Figure 10. Composite winds $\left(\mathrm{m} \mathrm{s}^{-1}\right)$ for the active monsoon days at $500 \mathrm{hPa}$ (left) and $200 \mathrm{hPa}$ (right) (a, d) ERA Interim (b, e) Zoom simulation (c, f) No-zoom simulation.

Figure 11. LPS tracks (a, b) and density maps of LPS (c, d). The left and right columns are for the zoom and no-zoom simulations respectively. The mean LPS track is shown by thick black line. LPS density is computed on $1^{\circ} \times 1^{\circ}$ grid boxes by counting the number of LPS passing through a given grid box.

Figure 12: Precipitation $\left(\mathrm{mm} \mathrm{day}^{-1}\right)$ and $850 \mathrm{hPa}$ streamlines averaged during a typical long lived depression case from a) Zoom and b) No-zoom simulation.

Figure 13 Time-series of the frequency count (FC) of moderate-to-heavy rainfall over the MT domain simulated by the LMDZ GCM (a) Zoom (b) No-zoom. The unit of FC in the zoom version is number of counts per $\mathrm{N}_{\mathrm{Z}}(=1500)$; and number of counts per $\mathrm{N}_{\mathrm{nz}}(=273)$.

Figure 14. The patterns generated by regressing the $850 \mathrm{hPa}$ winds on the index of frequency count (FC) of moderate-to-heavy rainfall (a) Zoom (b) No-zoom. Unit of regression is $\mathrm{ms}^{-1}$ (std.dev FC) ${ }^{-1}$. 
a)
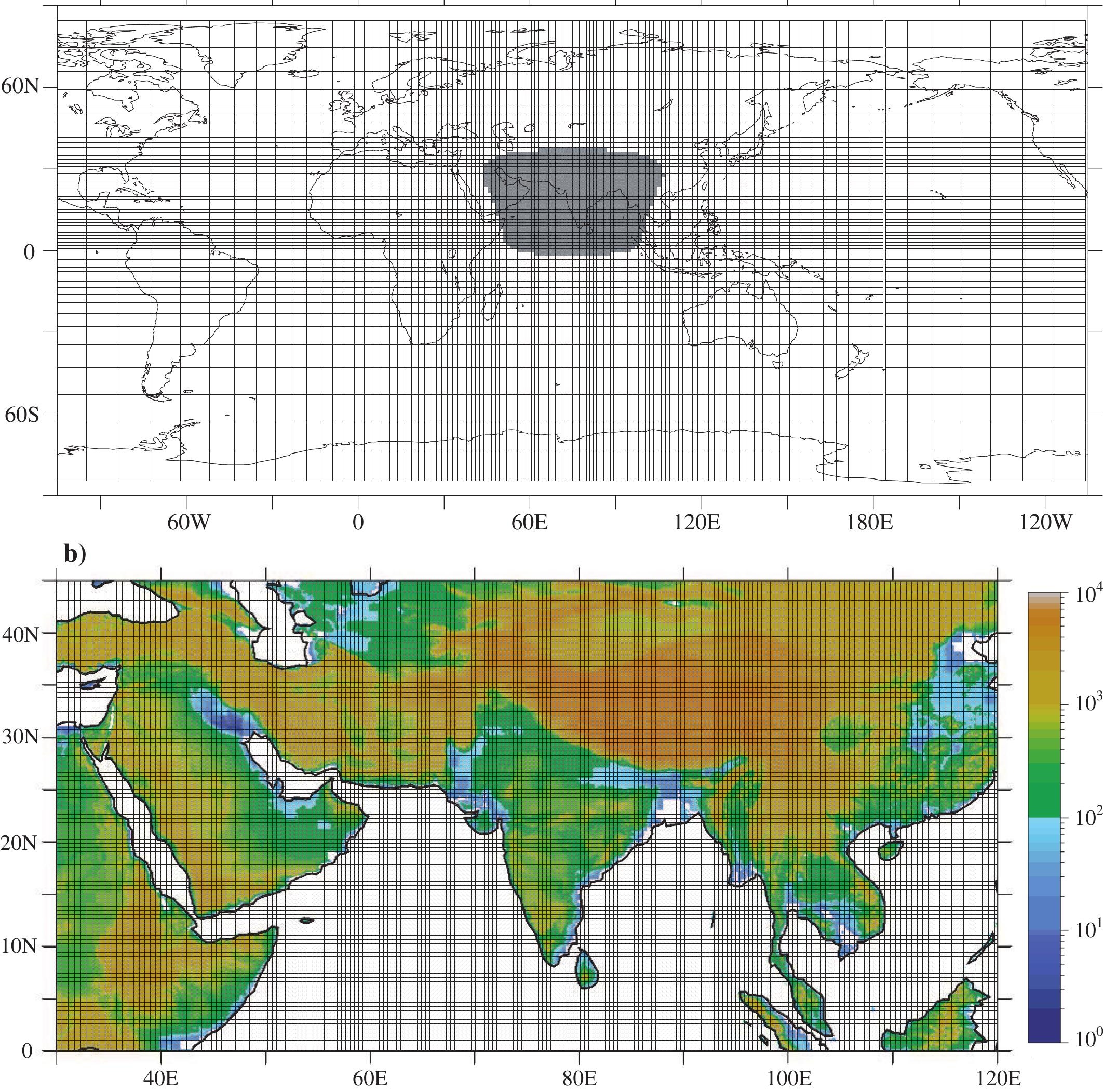
a) Mean winds at $850 \mathrm{hPa}(\mathrm{JJAS})$ - Zoom

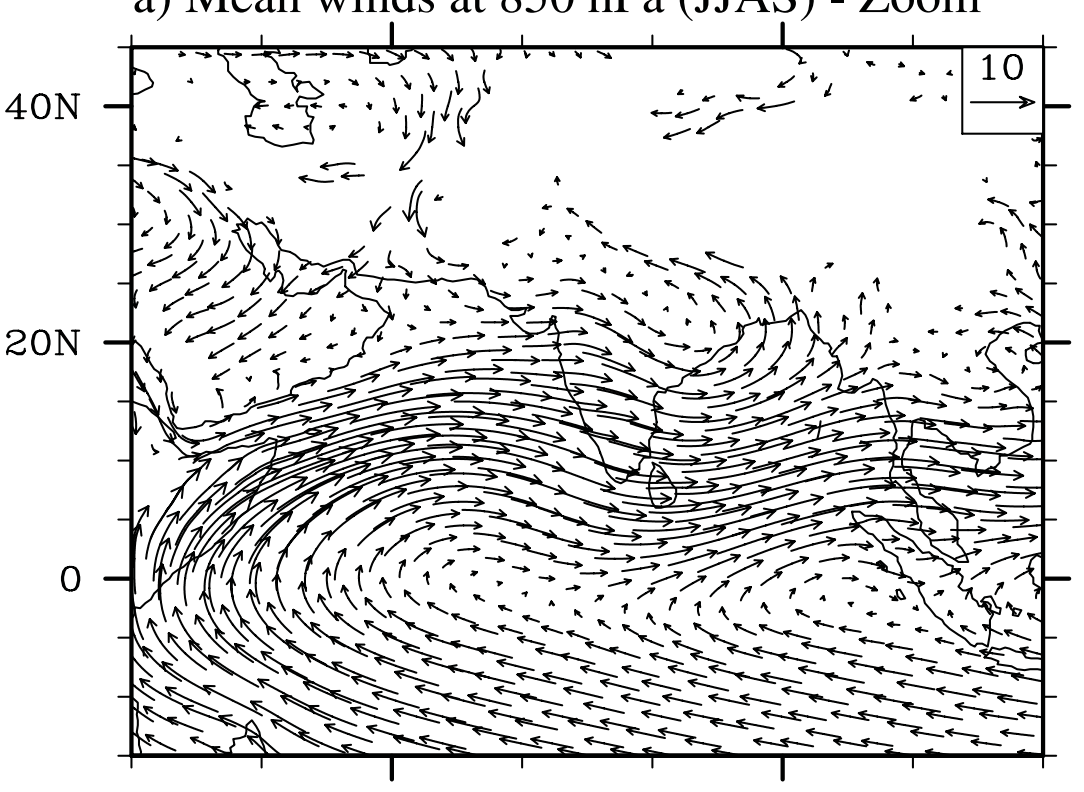

b) Mean Precipitation (JJAS) - Zoom

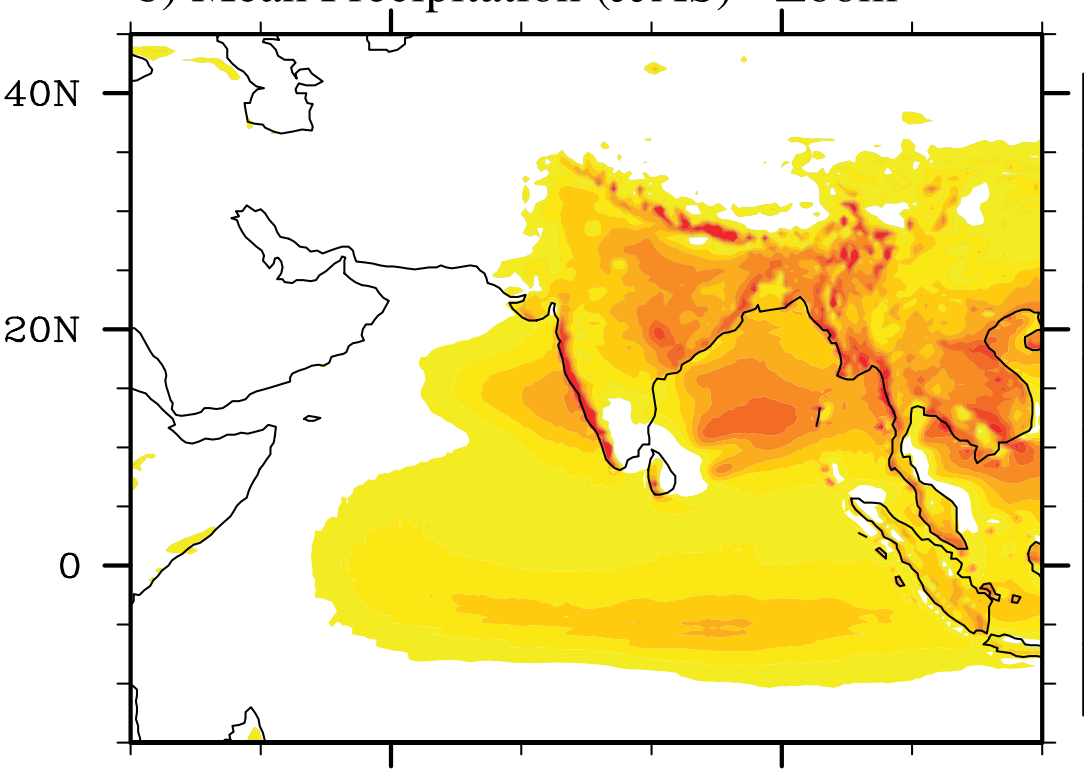

c) Rhum at $500 \mathrm{hPa}(\mathrm{JJAS})$ - Zoom

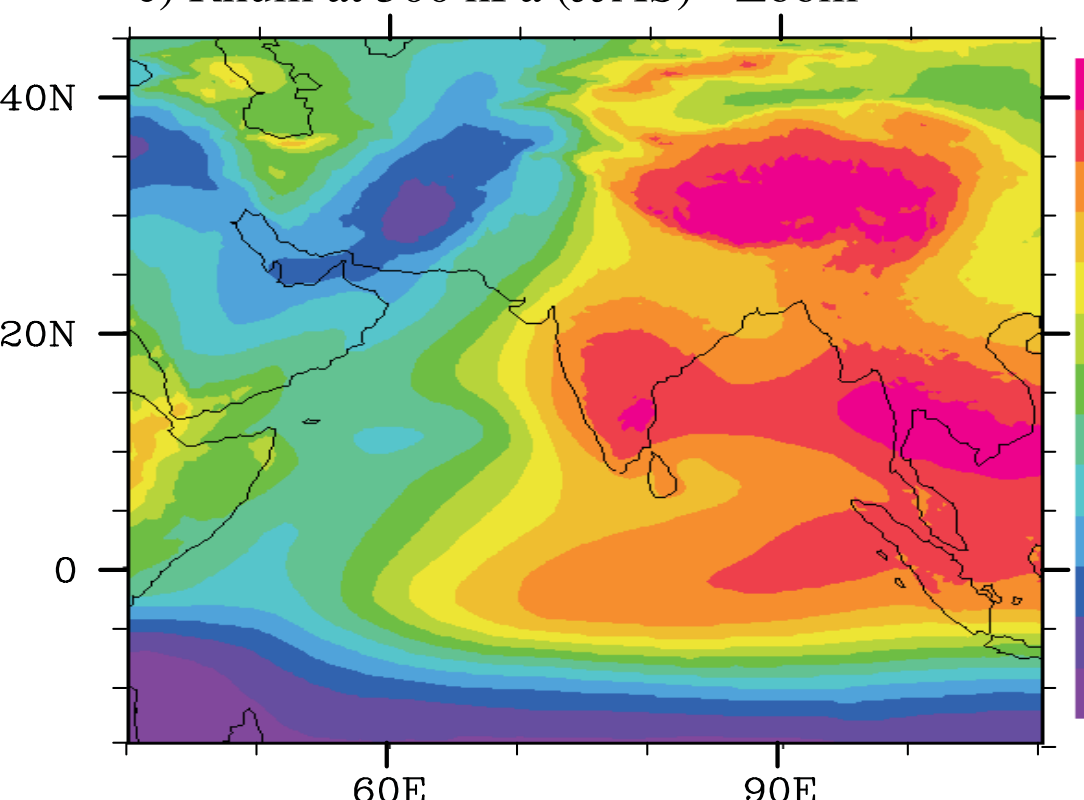

d) Mean winds at $850 \mathrm{hPa}$ (JJAS) - No Zoom

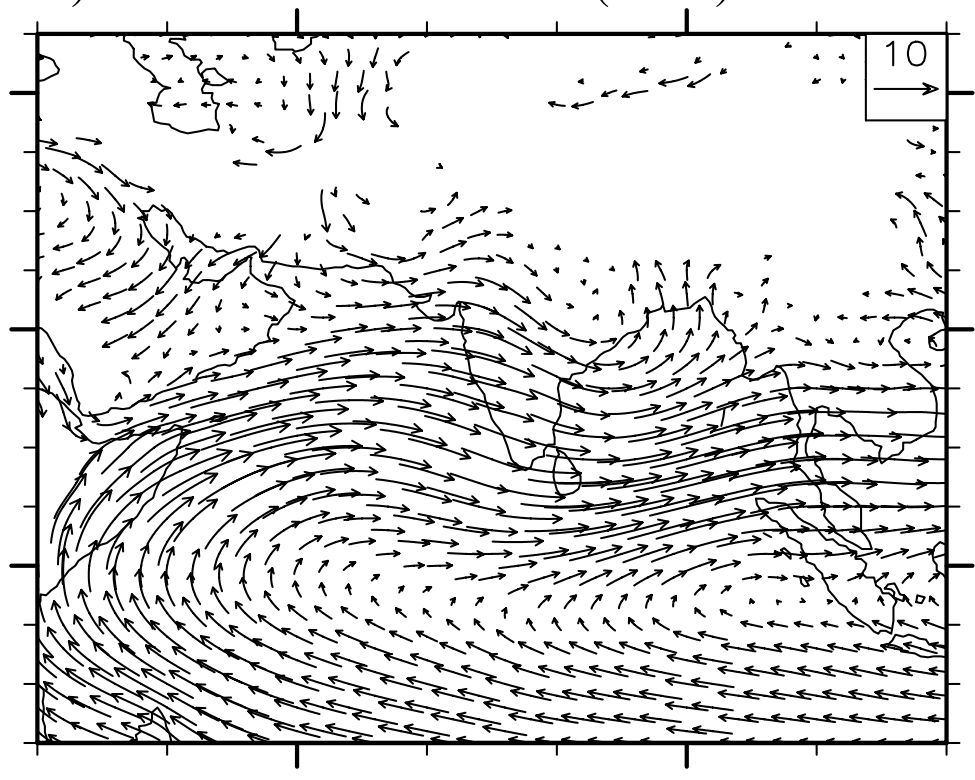

e) Mean Precipitation (JJAS) - No Zoom

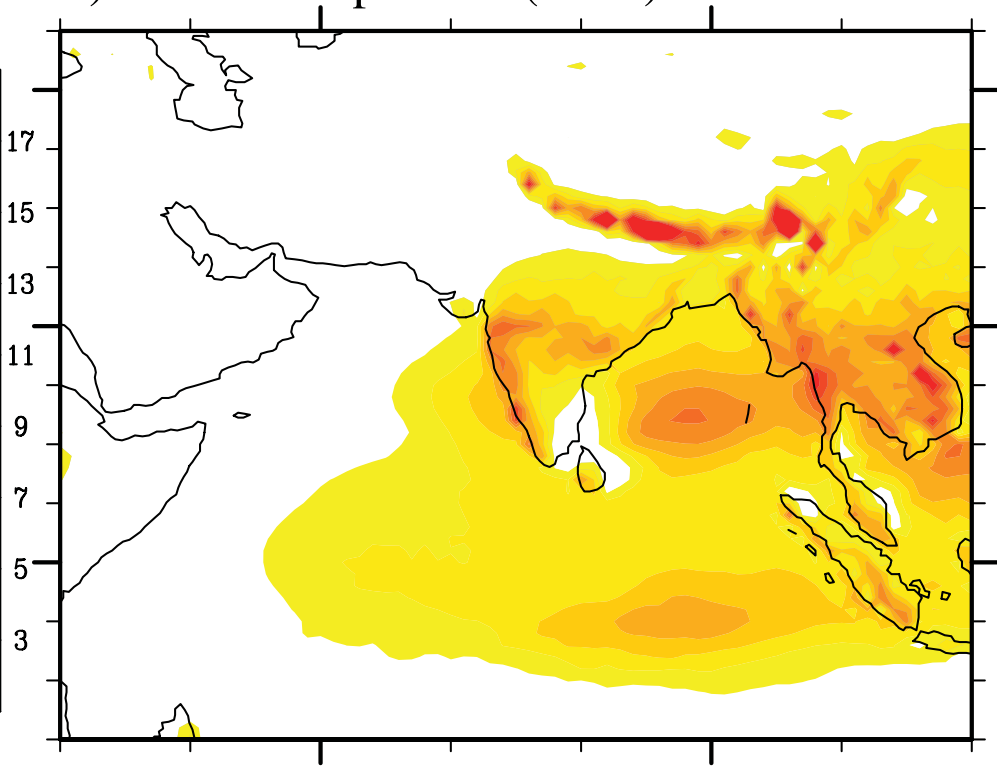

f) Rhum at $500 \mathrm{hPa}$ (JJAS) - No Zoom

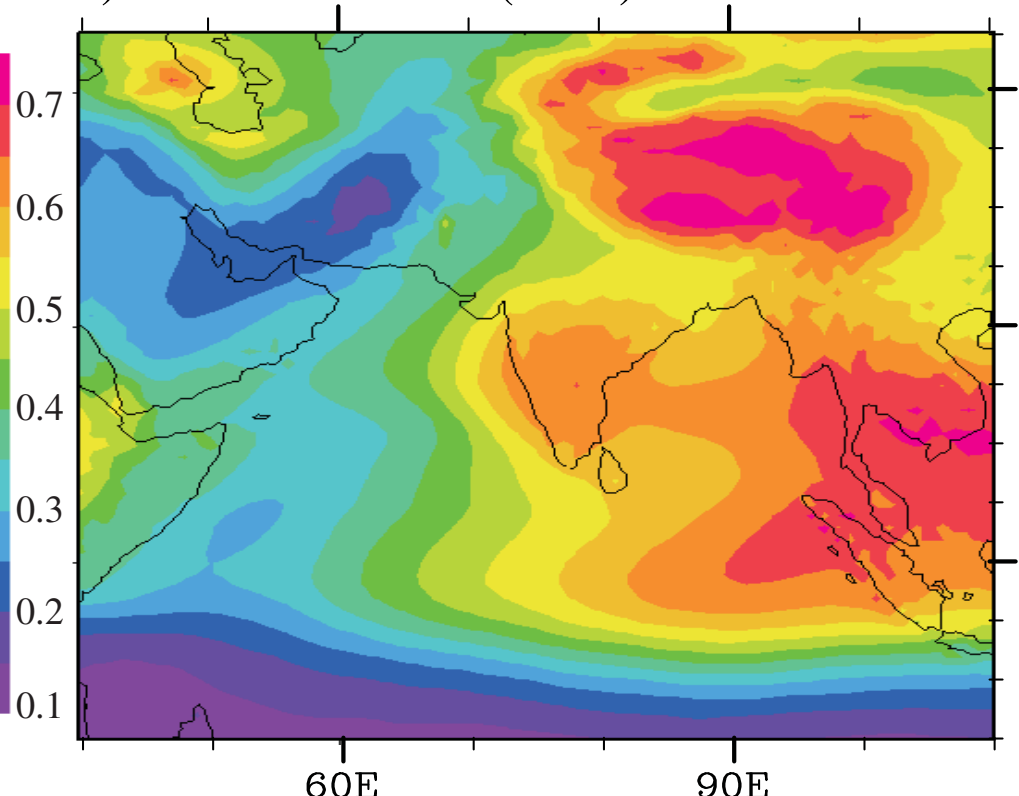




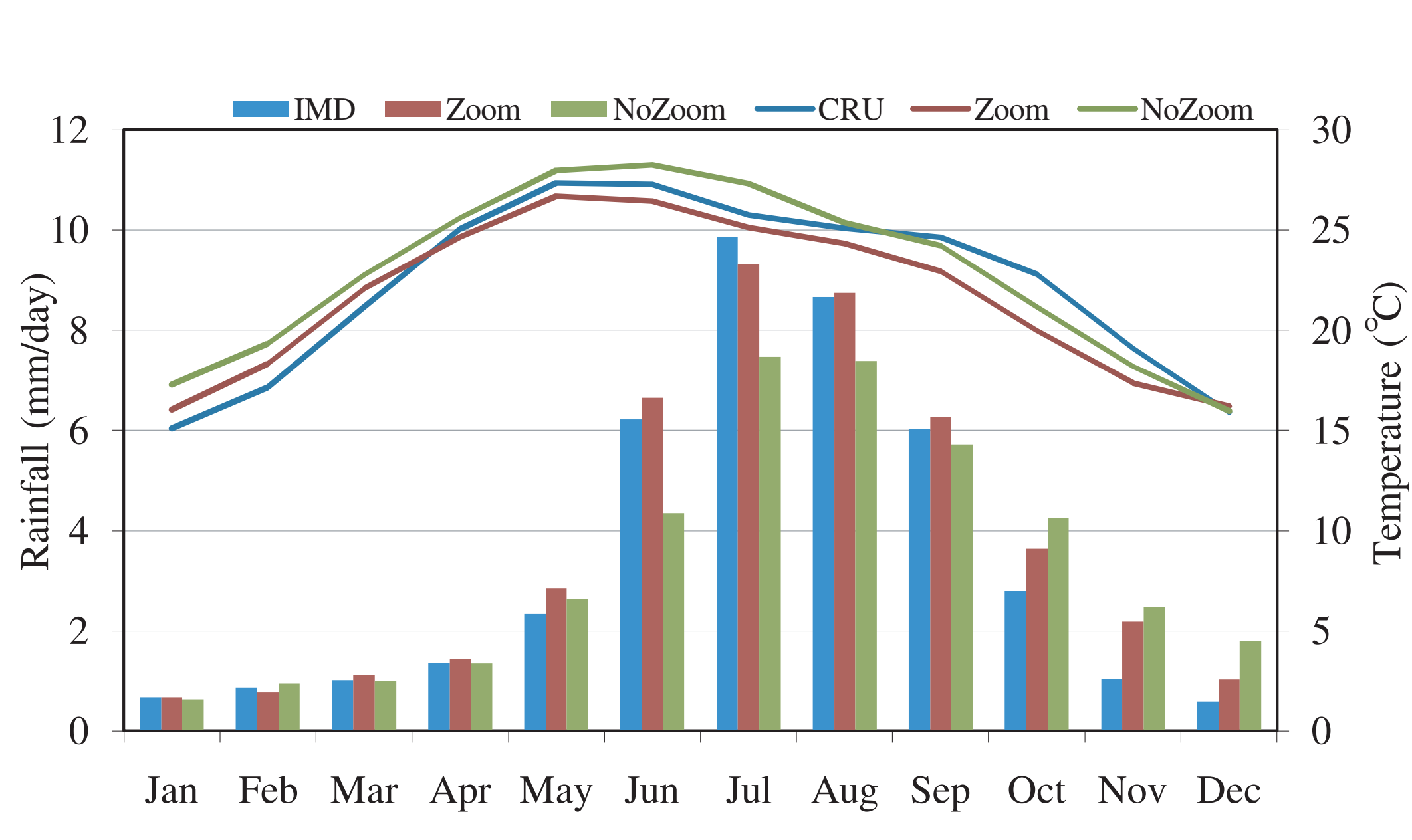

Jan Feb Mar Apr May Jun Jul Aug Sep Oct Nov Dec

\section{Figure-6}

(1) 
d) Relative Vorticity $\left(\times 10^{5}\right)$

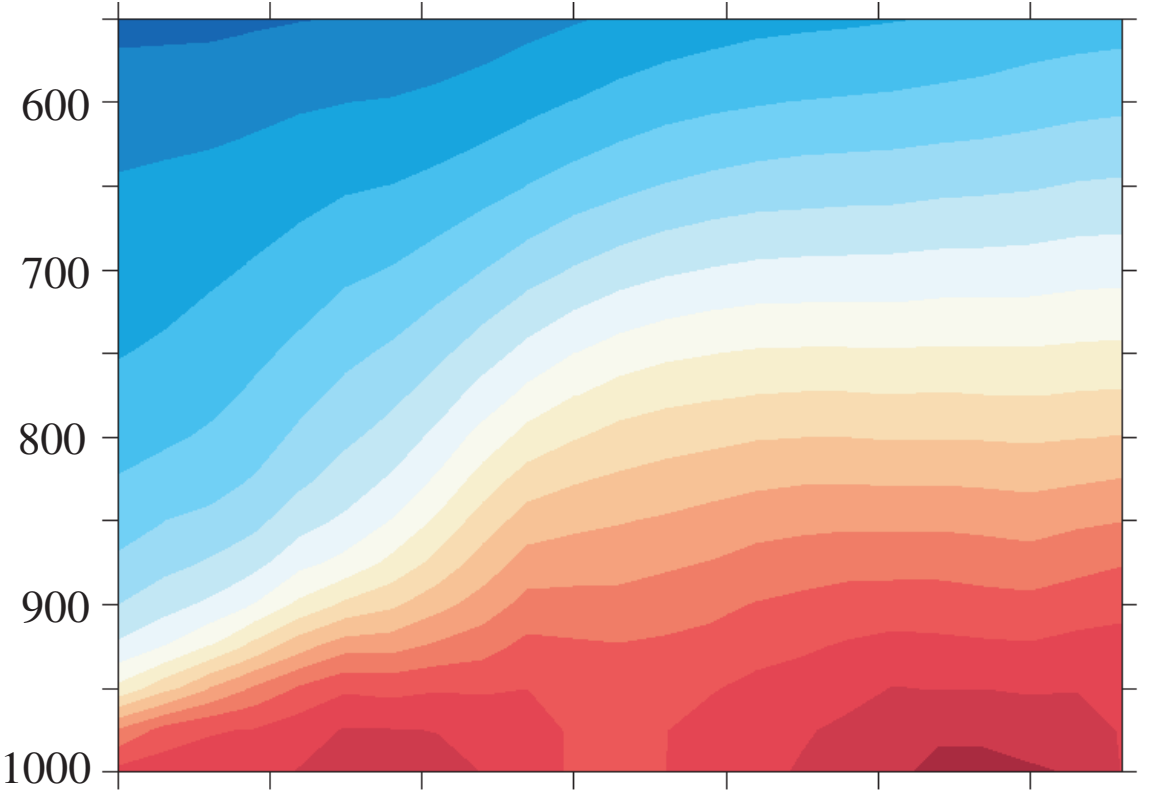

b) Specific Humidity (JJAS) - Zoom

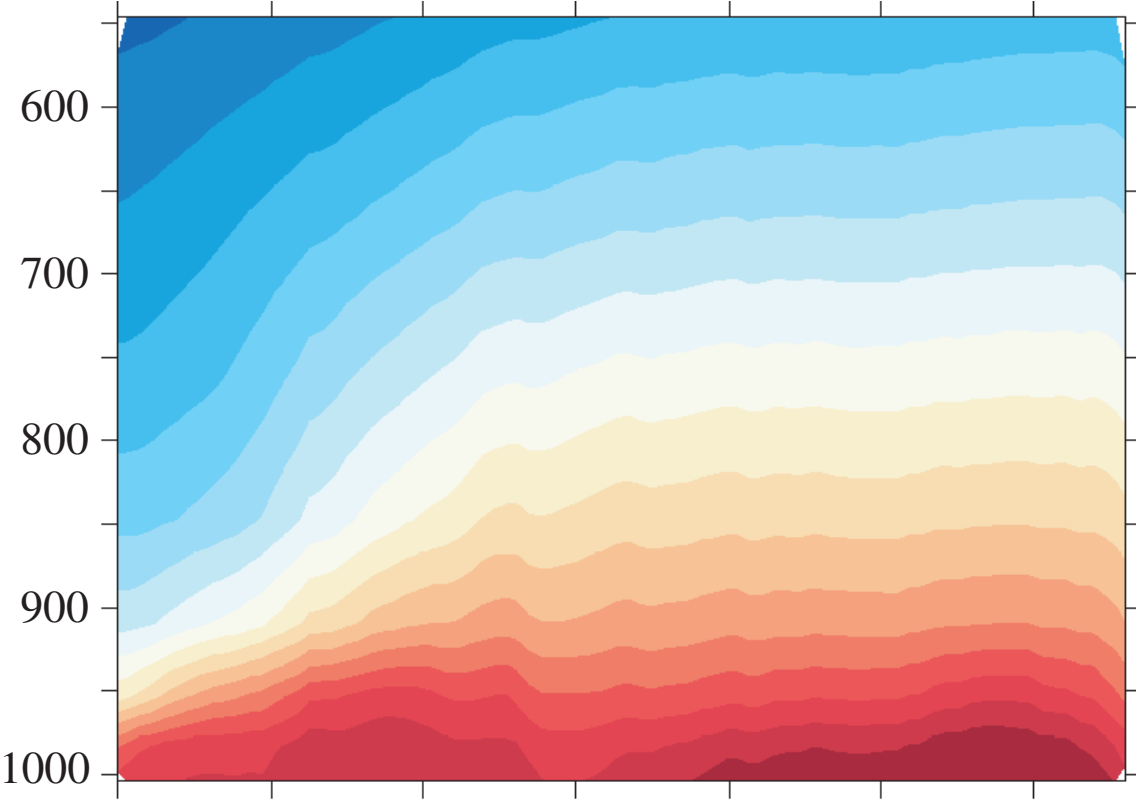

c) Specific Humidity (JJAS) - No Zoom

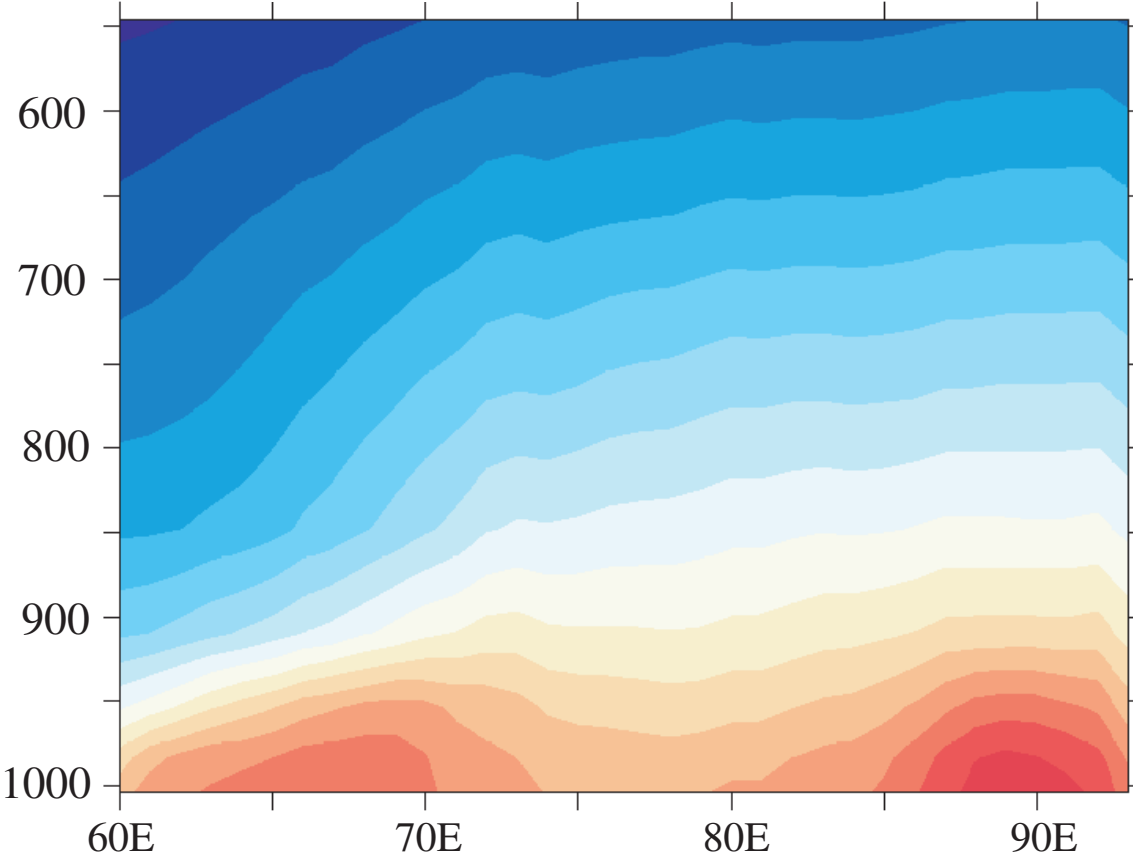

100
200
300
400
500
600
700
800
900
1000

$\begin{array}{ccc}-30 & -20 & -10 \\ \text { e) } & \text { Divergence } & (\mathbf{x} 10 \\ \end{array}$
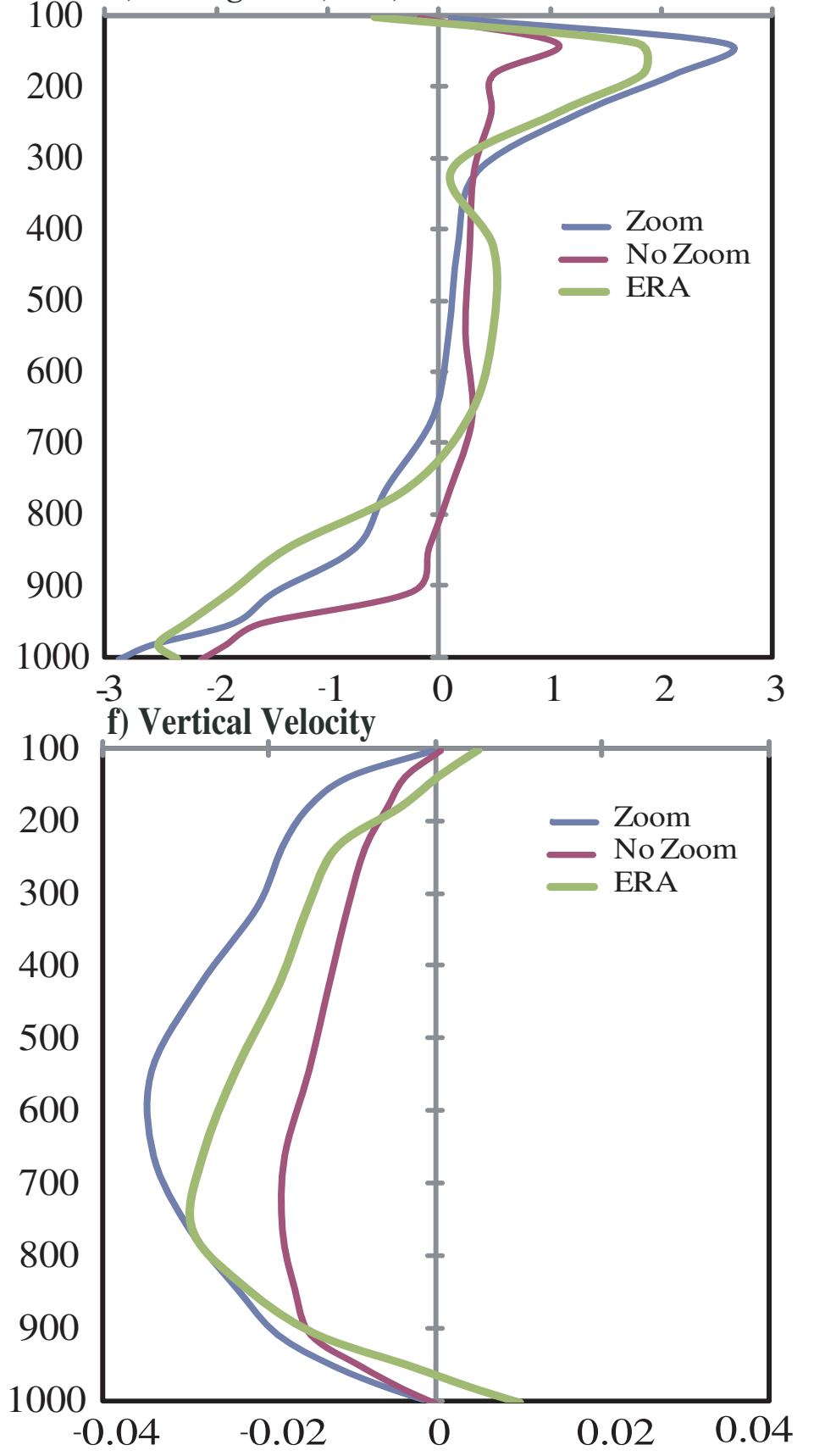

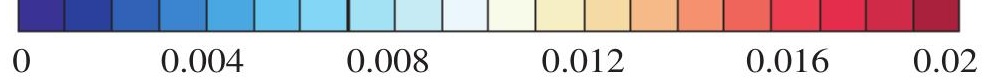


a) Mean Precipitation (Active) - IMD

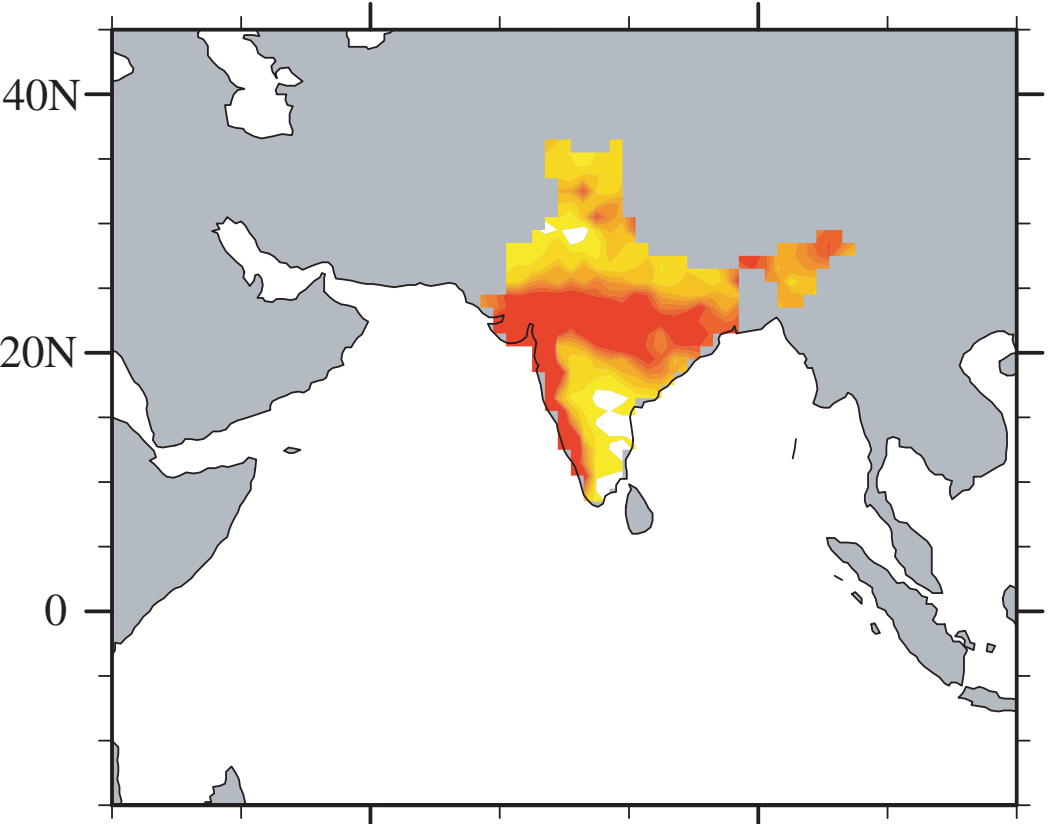

b) Mean Precipitation (Active) - Zoom

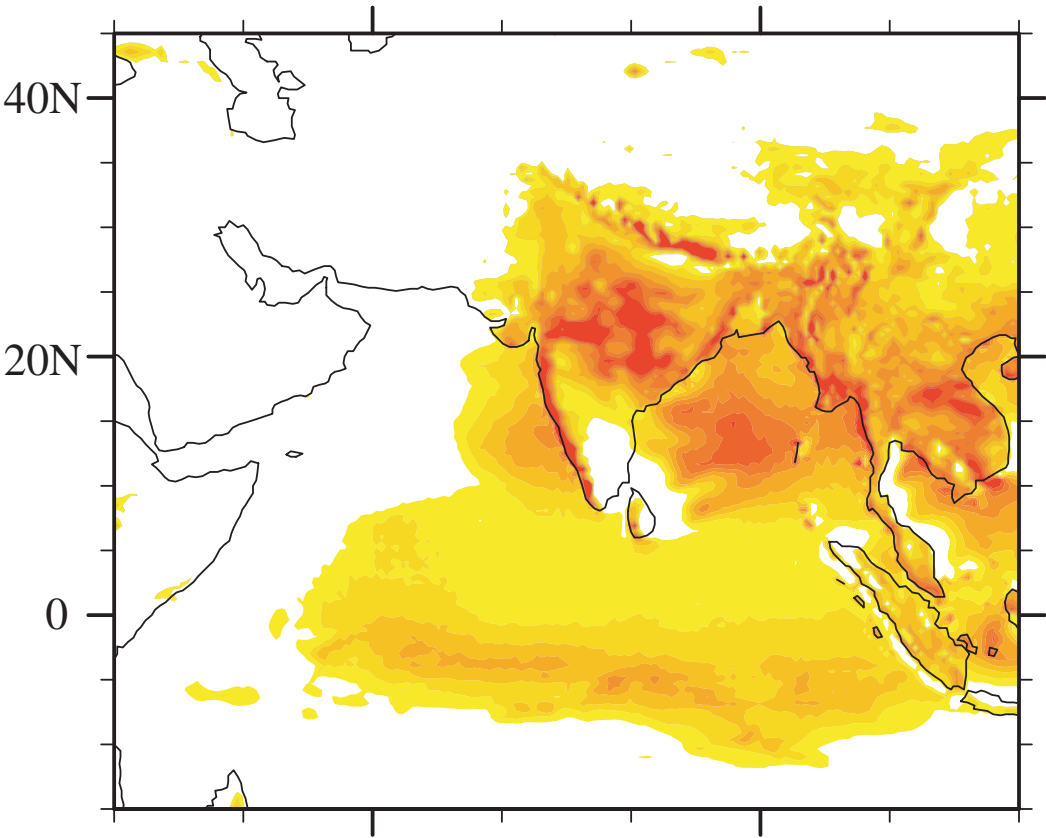

c) Mean Precipitation (Active) - No Zoom

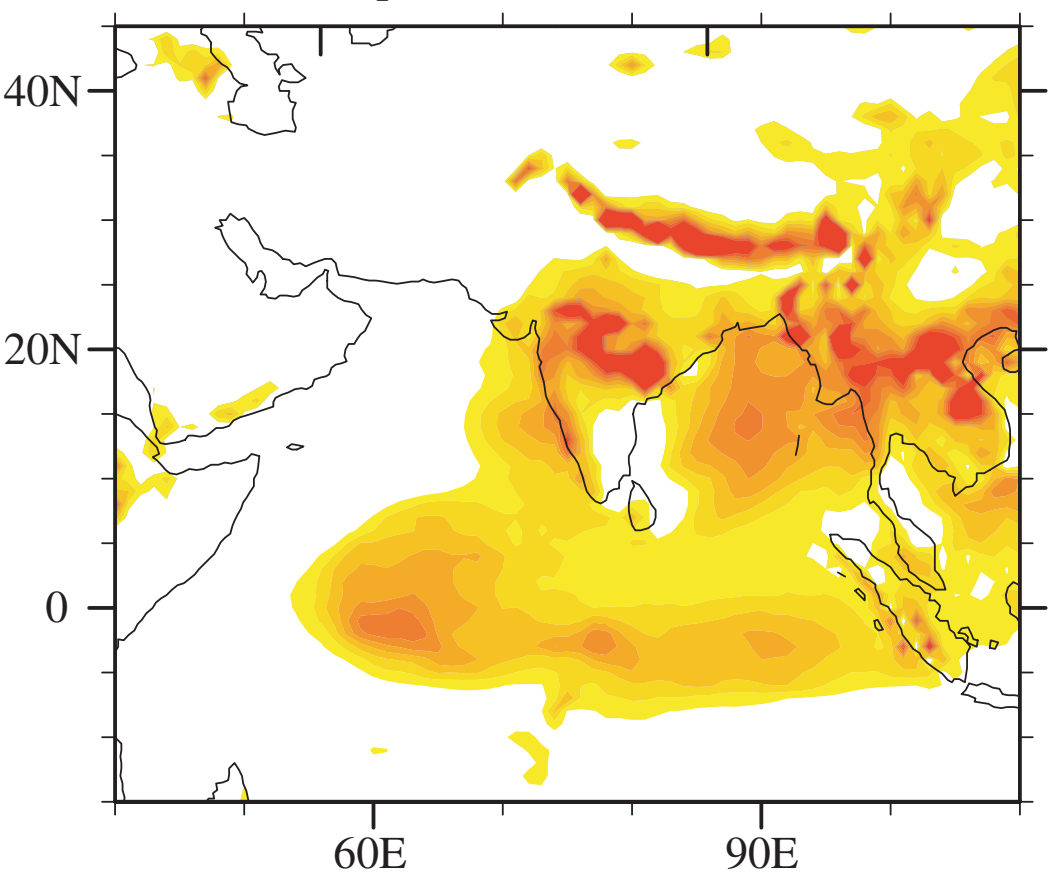

d) Mean wind at $850 \mathrm{hPa}$ (Active) - ERA

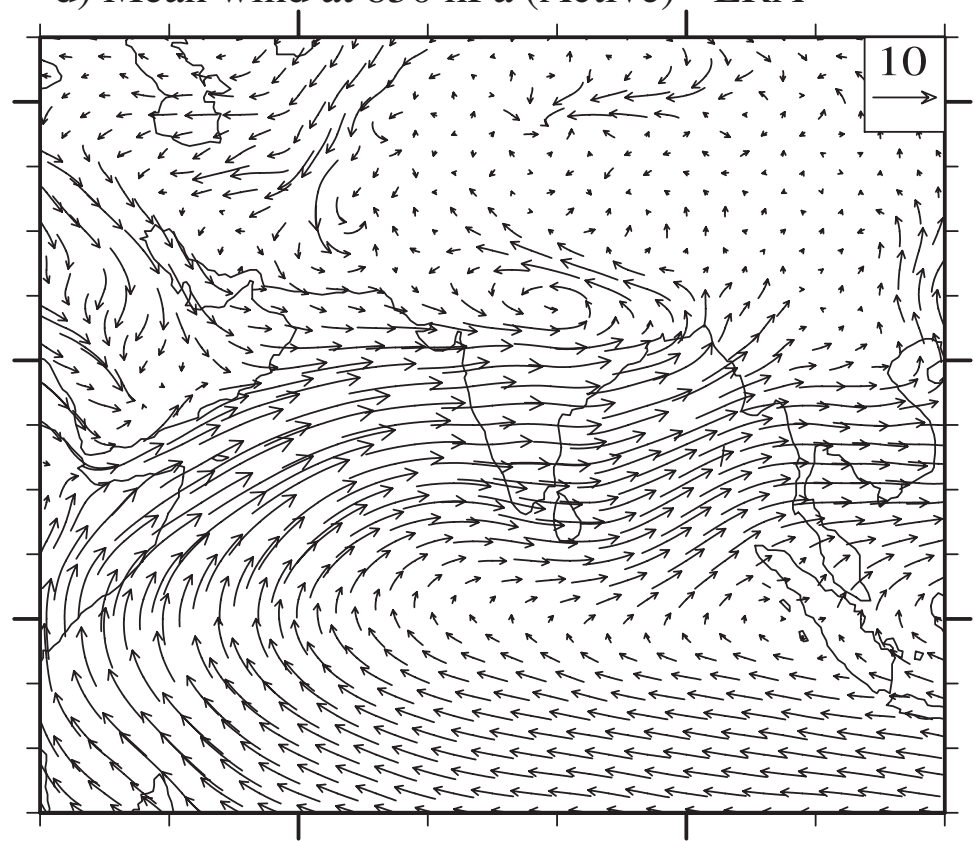

e) Mean wind at $850 \mathrm{hPa}$ (Active) - Zoom

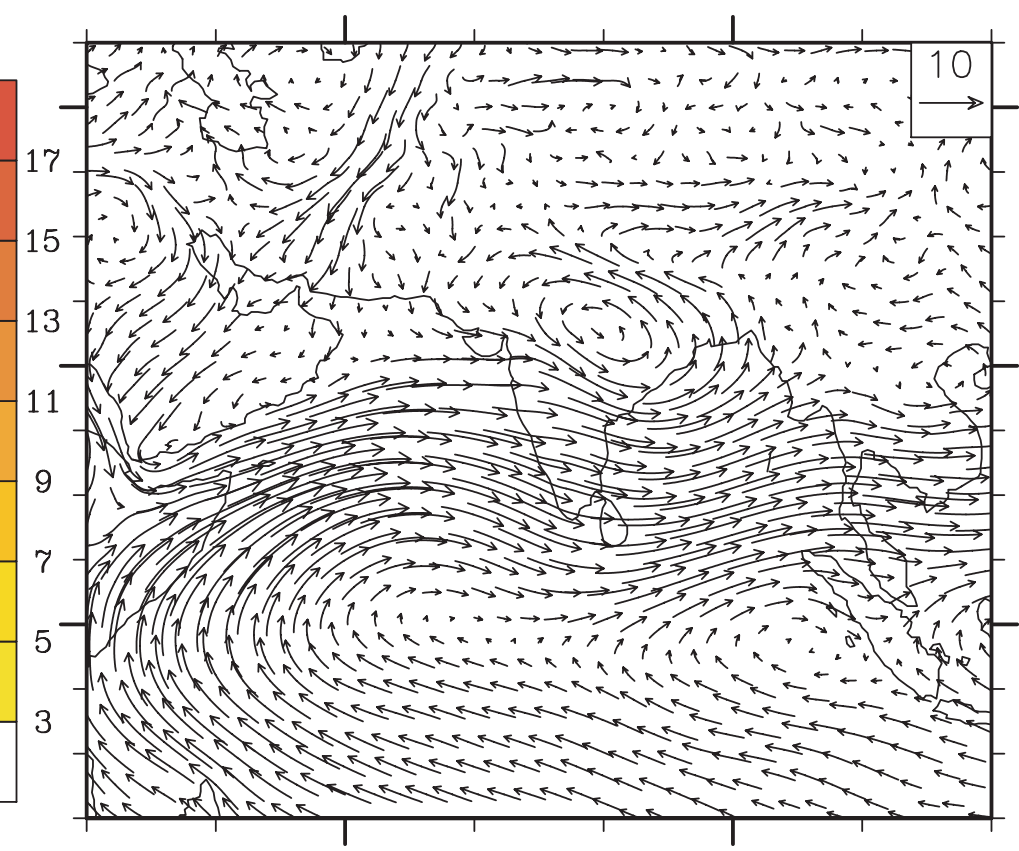

f) Mean wind at $850 \mathrm{hPa}$ (Active) - No Zoom

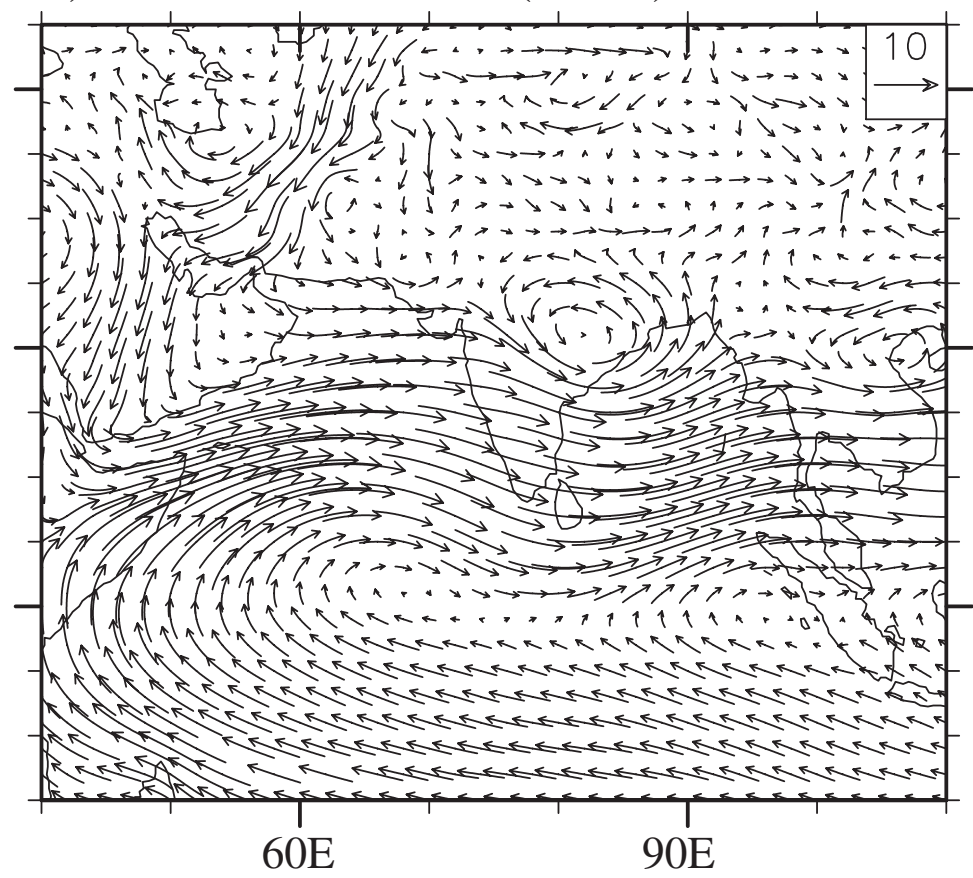


Figure-199 Wind at $500 \mathrm{hPa}(\mathrm{JJAS})$ - ERA

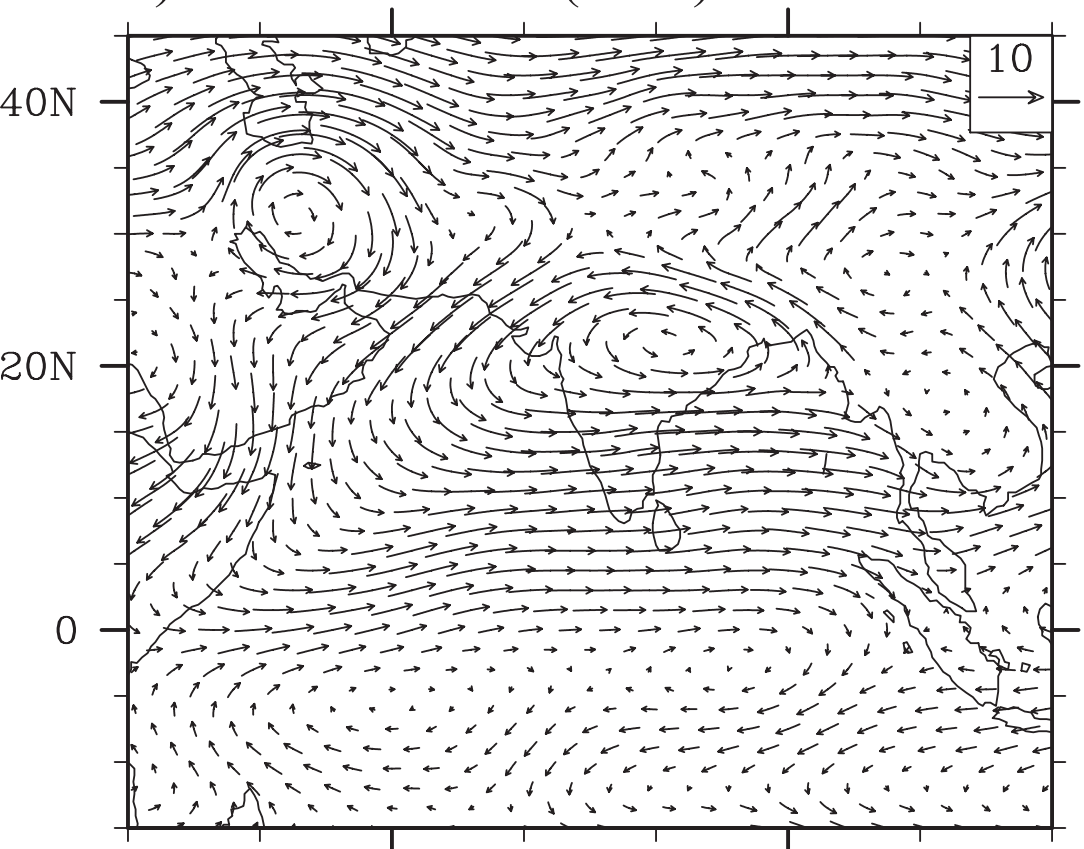

b) Wind at $500 \mathrm{hPa}(\mathrm{JJAS})$ - Zoom

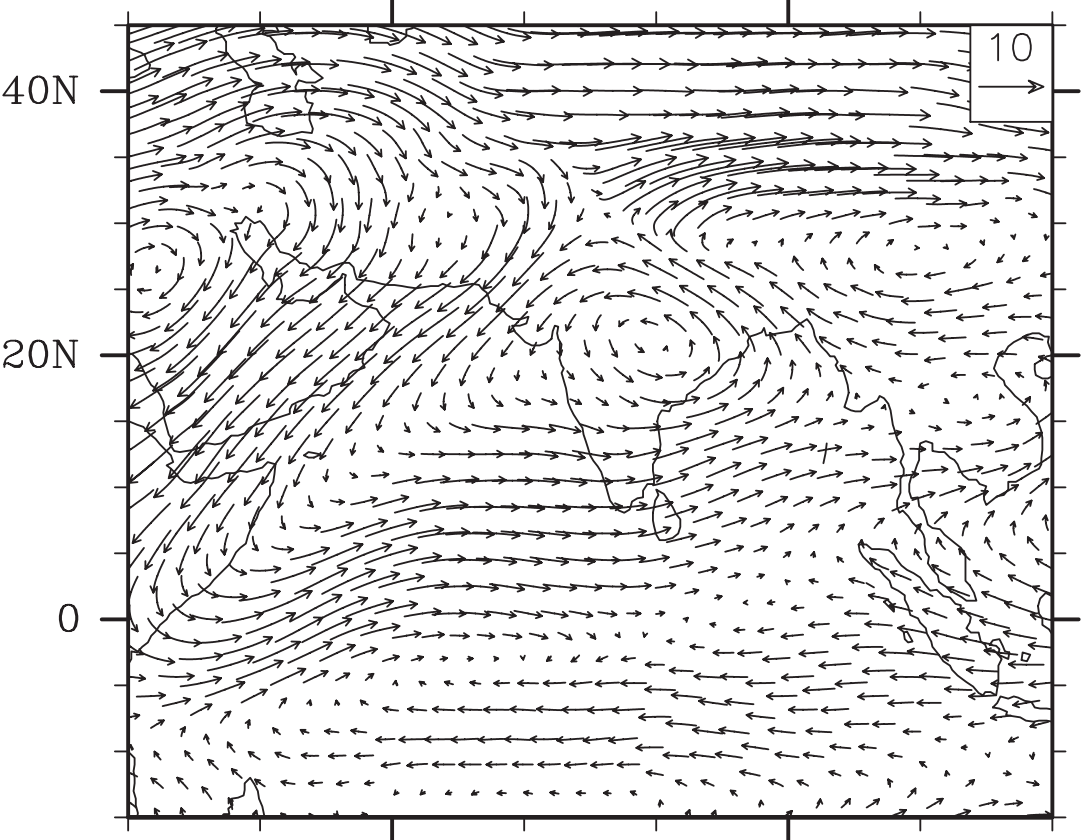

c) Wind at $500 \mathrm{hPa}$ (JJAS) - No Zoom

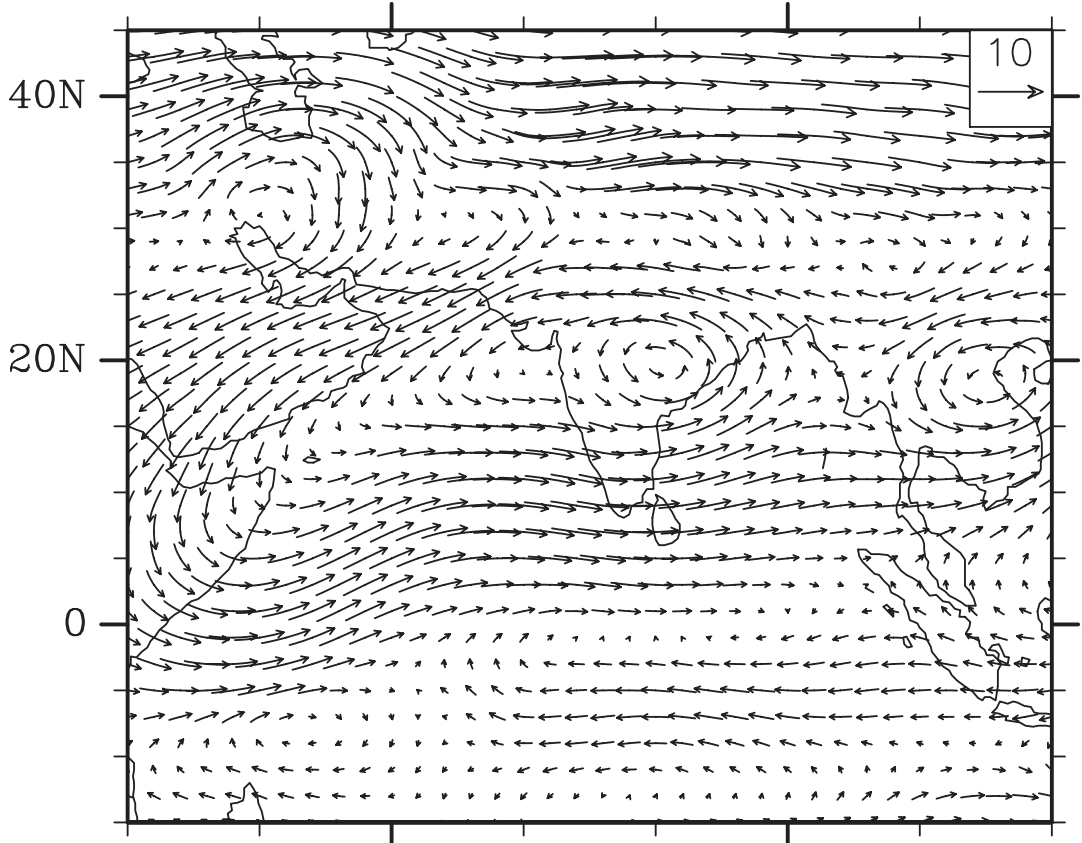

$60 E$ d) Wind at $200 \mathrm{hPa}$ (JJAS) - ERA

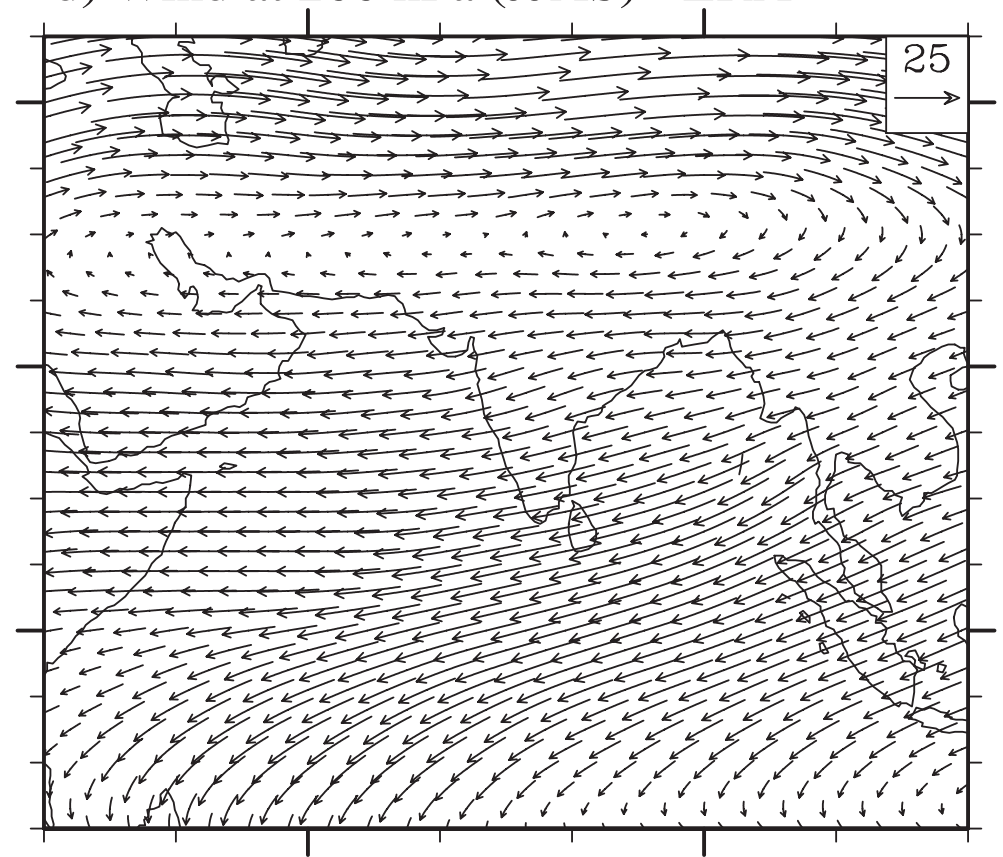

e) Wind at $200 \mathrm{hPa}(\mathrm{JJAS})$ - Zoom

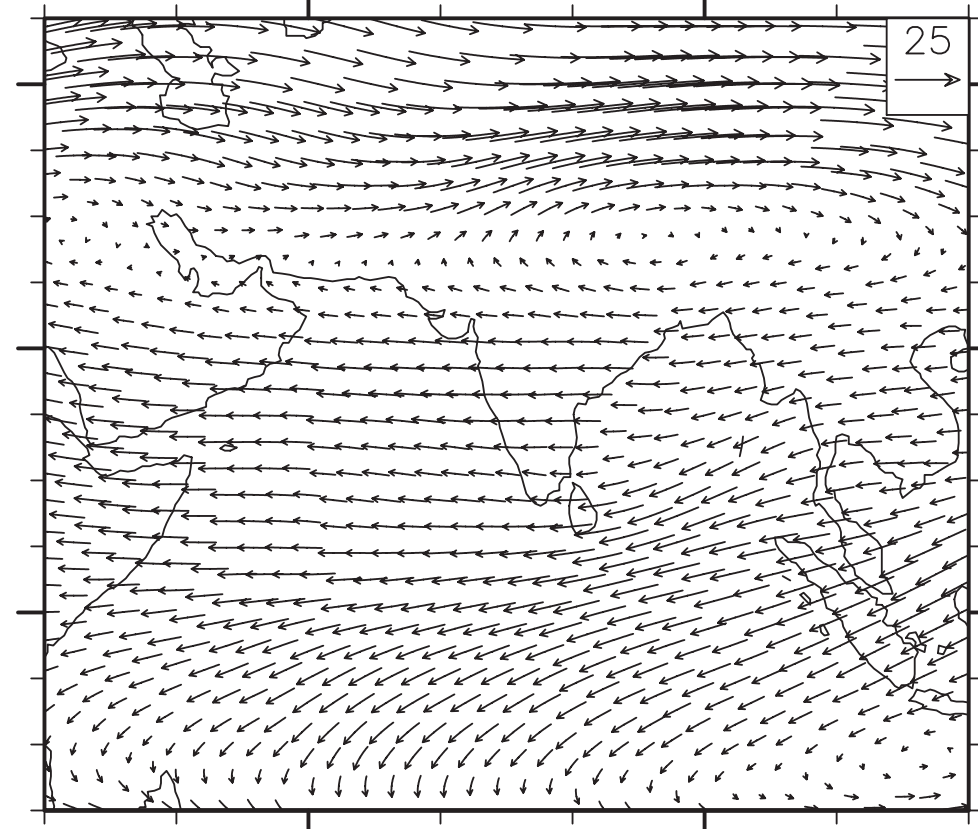

f) Wind at $200 \mathrm{hPa}(\mathrm{JJAS})$ - No Zoom

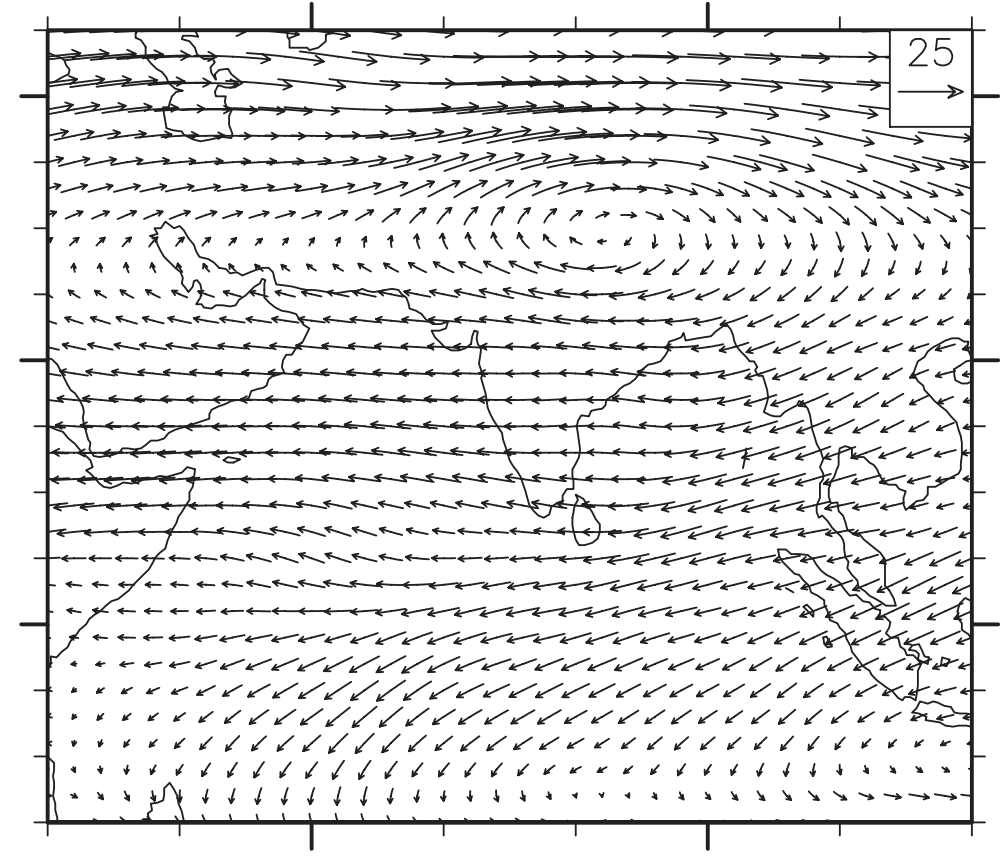


a)

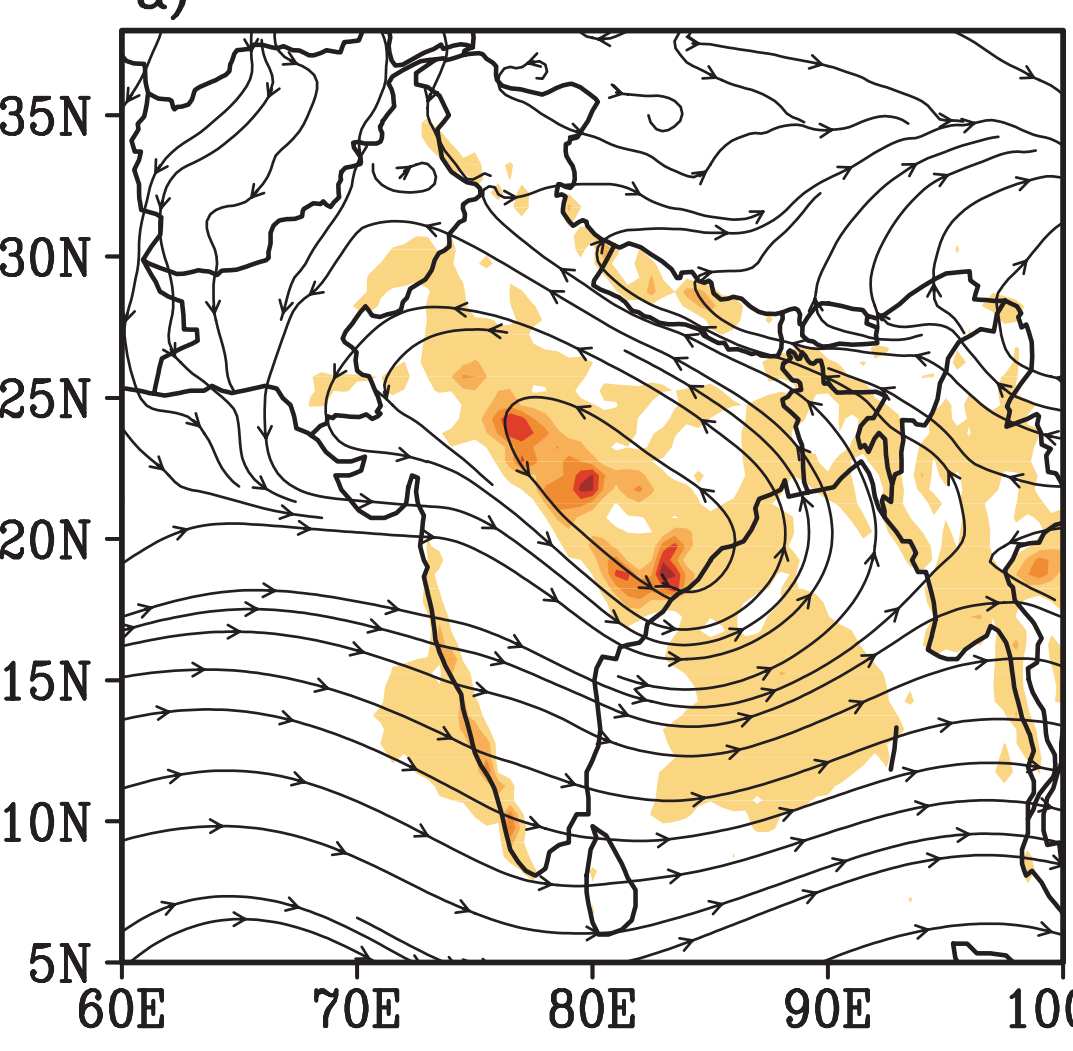

b)

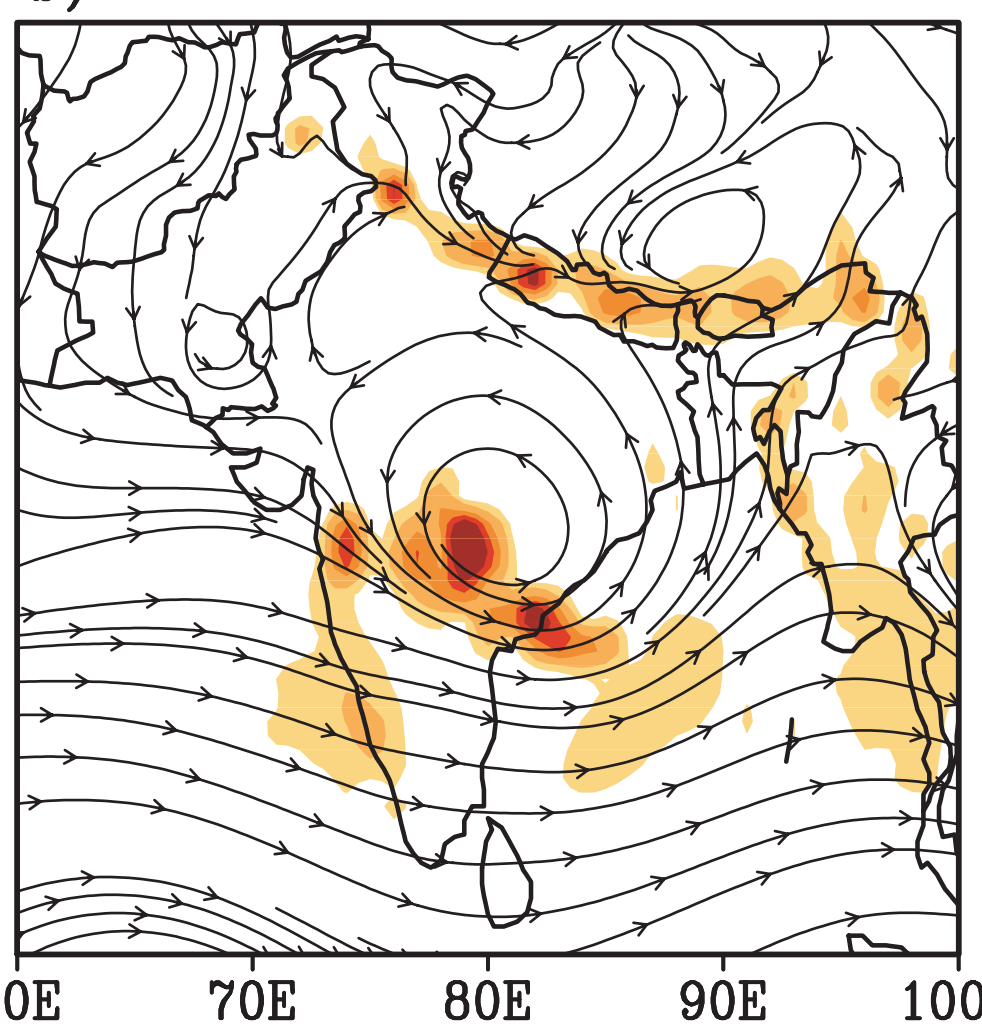




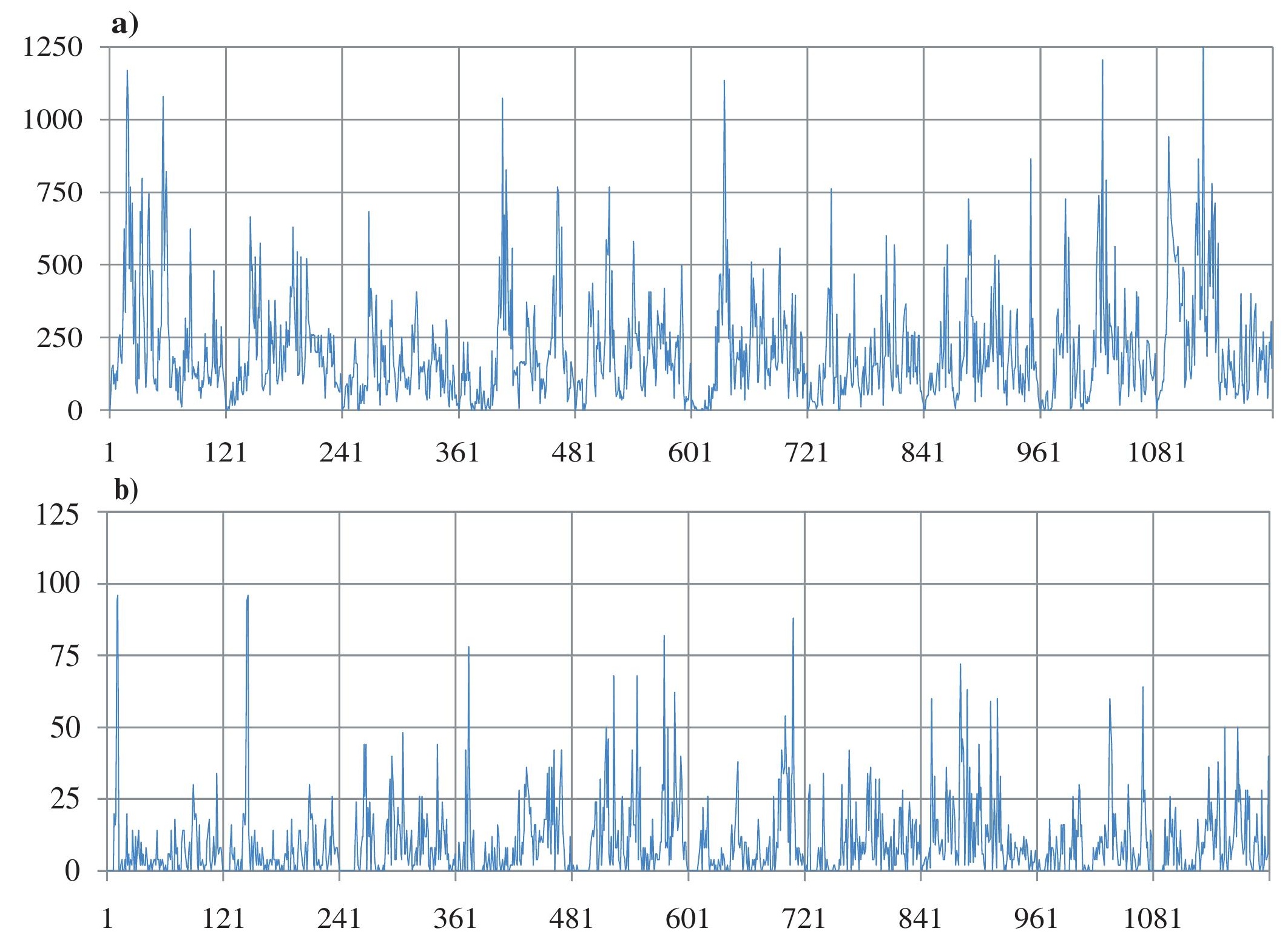


a)

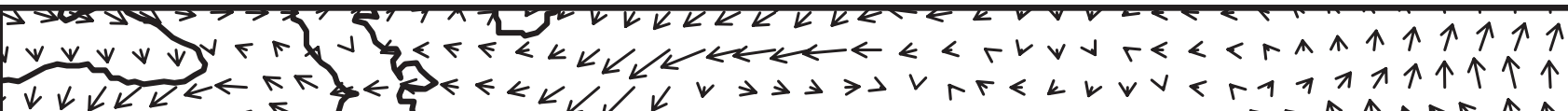

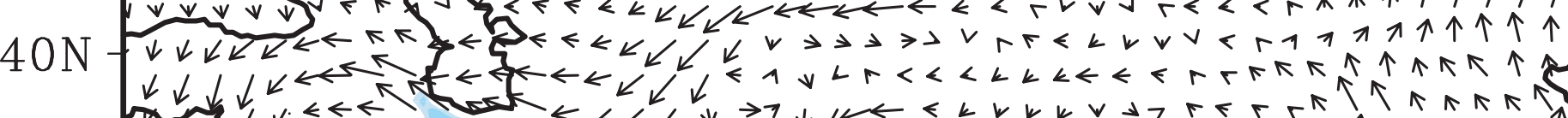

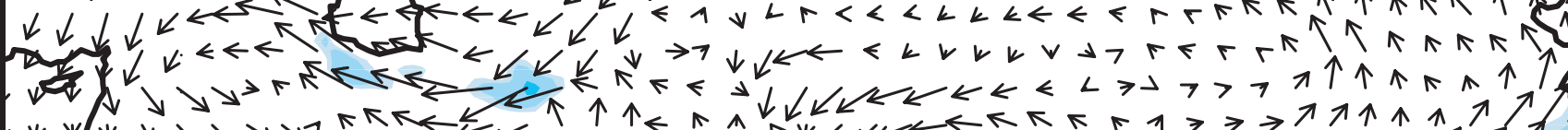

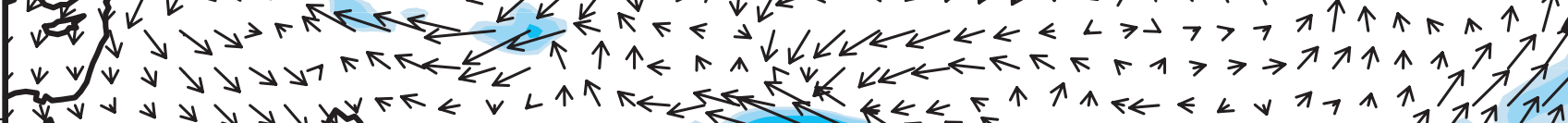

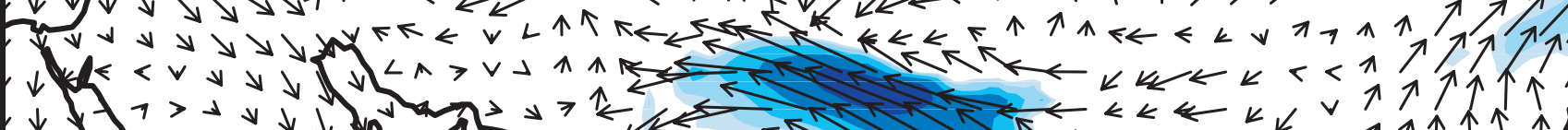

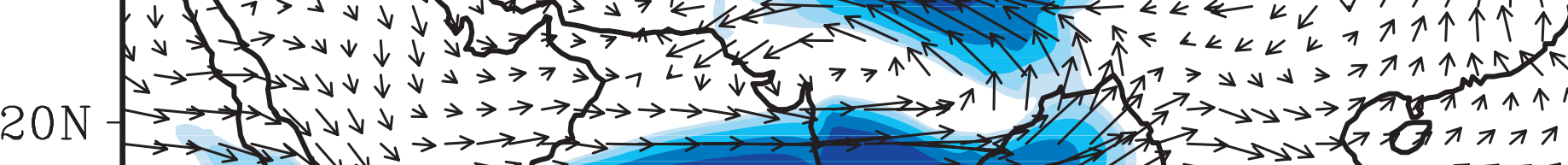

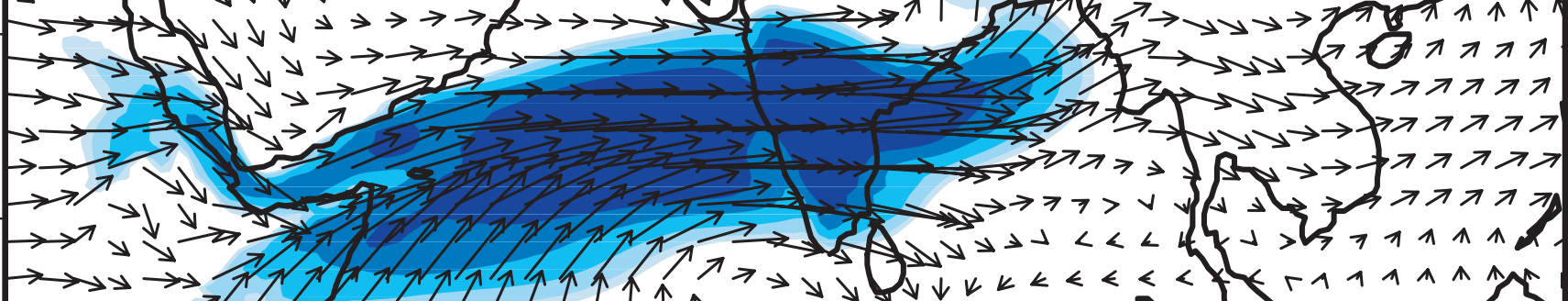

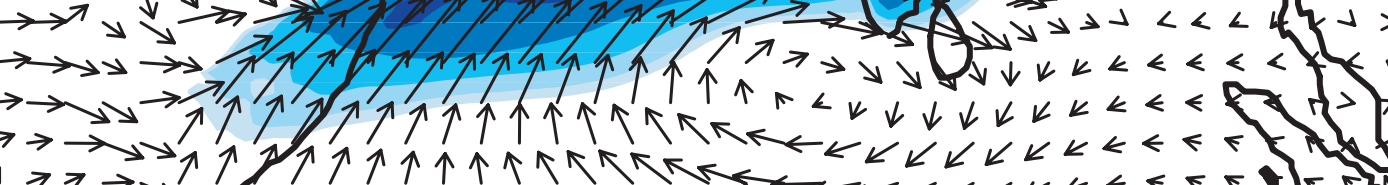

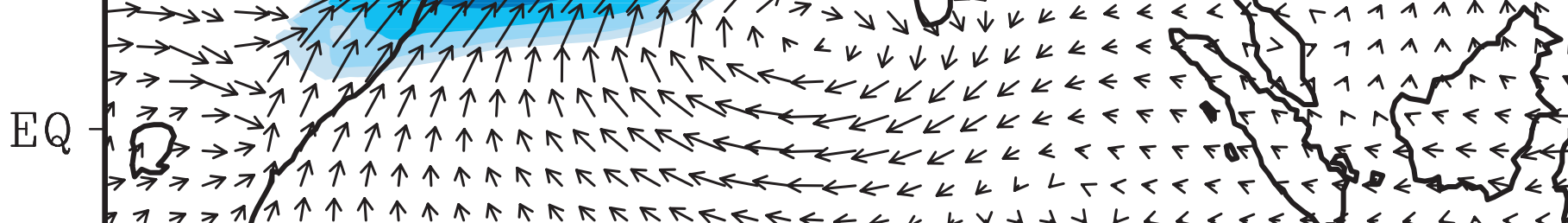

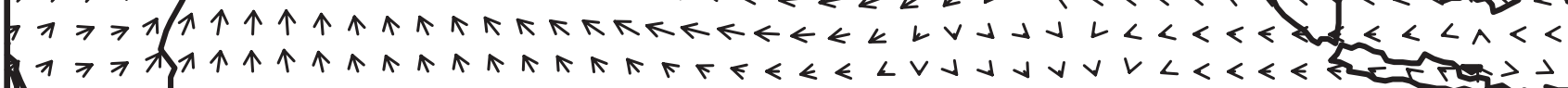

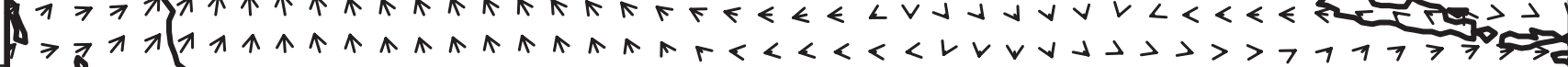

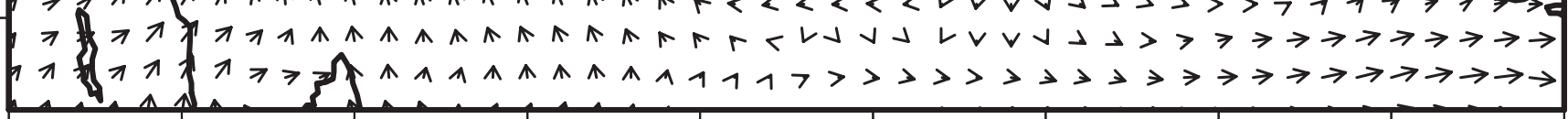
b)

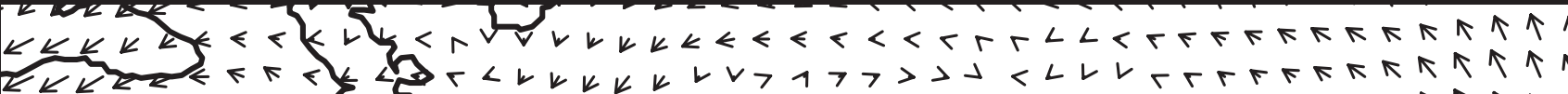
$40 \mathrm{~N}$

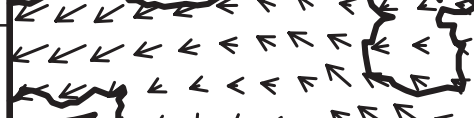
$<<$

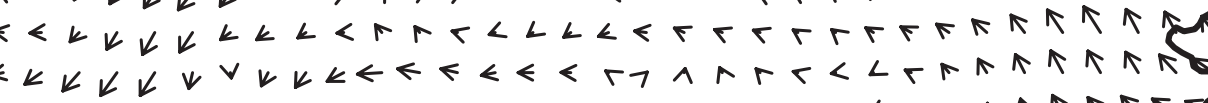

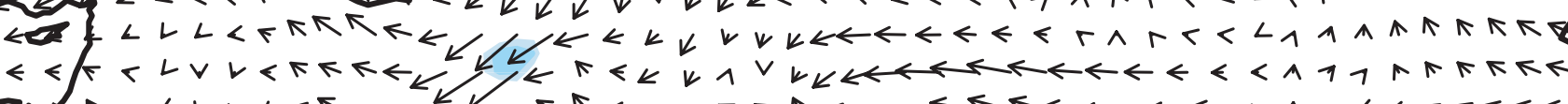

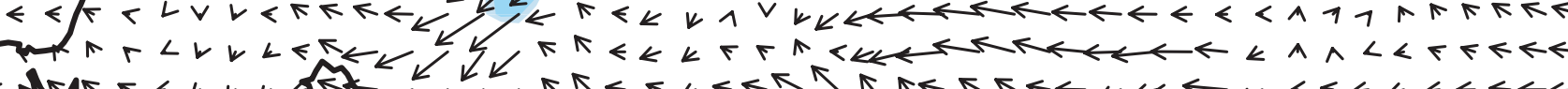
Arg $r<k k k$ \& f

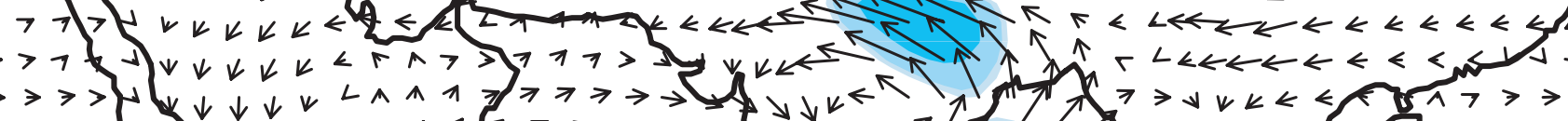

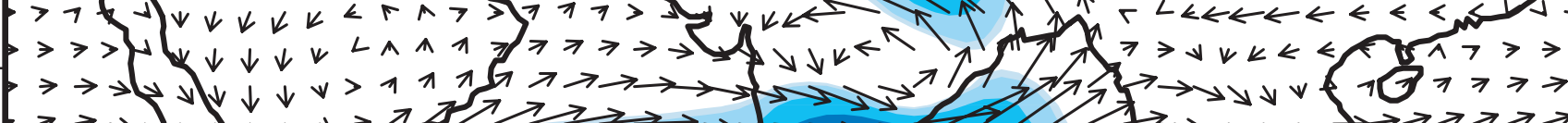

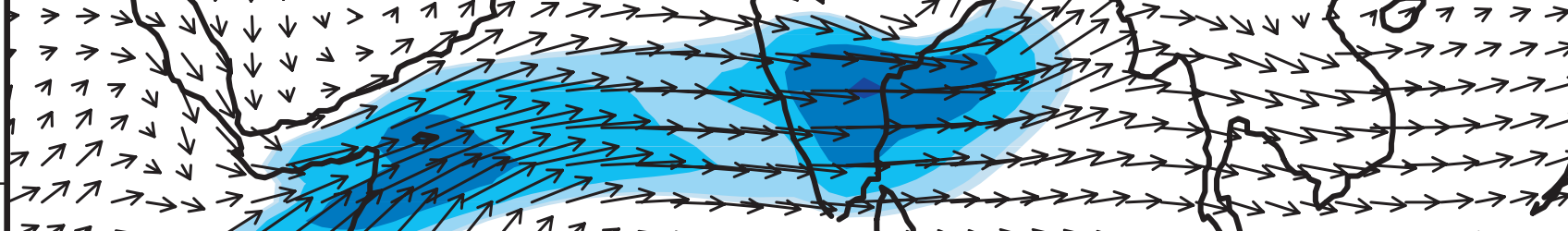

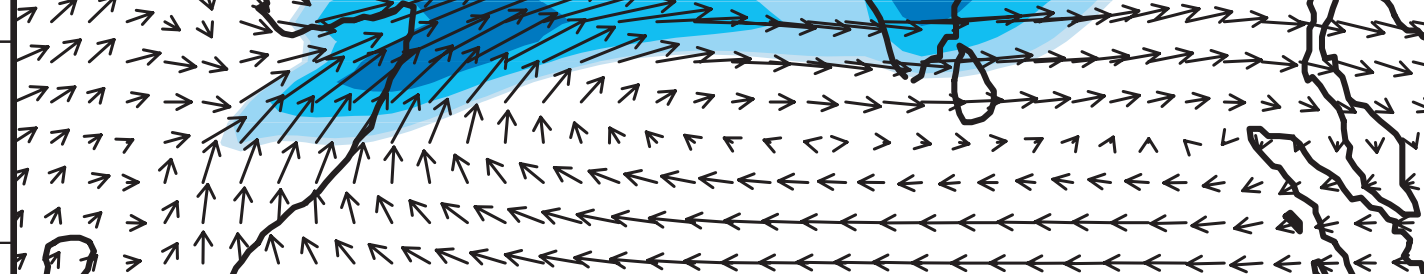
pin

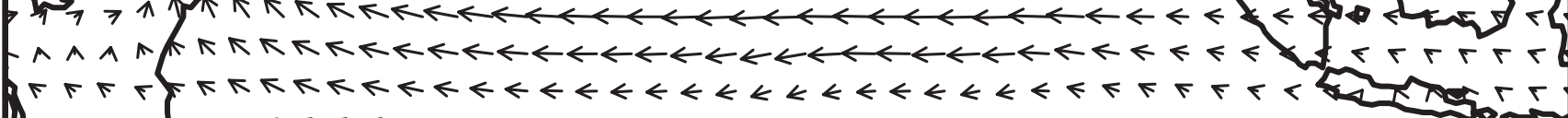

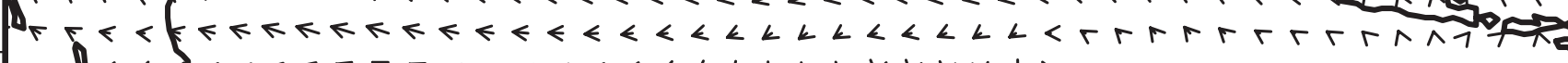
$<f<<\}<<<F R F F<<<<<<L L レ \vee \vee \vee \vee \vee \vee V>>7 \wedge \wedge \wedge \wedge \wedge \wedge \wedge \wedge \wedge \wedge 7>$

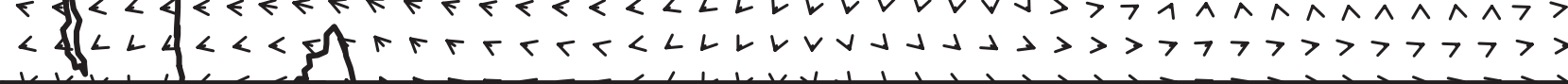
40E
$60 \mathrm{~F}$ 